\title{
SECOND ORDER HOMOGENIZATION OF SUBWAVELENGTH STRATIFIED MEDIA INCLUDING FINITE SIZE EFFECT
}

\author{
JEAN-JACQUES MARIGO* AND AGNÈS MAUREL ${ }^{\dagger}$
}

Résumé. We present a homogenization method to find the effective behavior of a periodically stratified slab which accounts for the finite size of the slab. The effective behavior is that of a homogeneous anisotropic slab associated with discontinuity conditions, or jump conditions, for the displacement and for the normal stress at the boundaries of the slab. The coefficients entering in the effective homogenized wave equation are related to the geometry and to the composition of the layers only, as in the classical homogenization. Those entering in the jump conditions are related to boundary layer effects and thus they depend also on the properties of the media surrounding the slab. The validity of our homogenization method is inspected in the case of layers associated with Neumann boundary conditions.

Key words. homogenization, two-scale method, matched asymptotic expansion, stratified media, finite size effects, effective jump conditions,

AMS subject classifications. 34E13, 35B27,74Q10, 74Q15,80M35, 74J20,

1. Introduction. The scattering properties of media stratified at a subwavelength scale are known to be correctly described by an equivalent homogeneous anisotropic medium whose effective bulk parameters involve averages of the bulk parameters of the layers (the parameters entering in the wave equations); for shear horizontal (SH) elastic waves, it is the mass density and the shear modulus, and the averages involve also the surface or volume fractions of the layers. This is known since Rytov's work in 1956 [14] and the result has been extended within a rigorous mathematical framework to periodic media using the homogenization theory, see e.g. [3].

In its classical form, the homogenization is performed considering that the structured medium occupies the whole space. Obviously in practice, samples of finite thickness $e$ are considered and it has been shown that, for small $e$, the scattering properties of the samples were not correctly described by their homogenized counterparts [7, 8, 9]. In this paper, we show that the homogenization theory can be adapted to stratified structures of finite thickness, which yields an equivalent slab whose scattering properties accurately describe those of the actual structure. We establish that, in addition to the bulk parameters entering in the effective wave equation, the homogenization makes interface parameters to appear, which enter in jump conditions at the boundaries of the equivalent slab (Figure 1). While the effective bulk parameters depend only on the characteristics of the structure at the microscale, the interface parameters result from boundary layer effects and as such, they depend also on the characteristics of the surrounding media.

The homogenization for finite size stratified structures is performed up to the second order in $\varepsilon \equiv k h \ll 1$ ( $k$ being the wavelength and $h$ the periodicity of the structure). It is presented in the section 2 and the approach essentially follows the one presented in [1] in the context of solid mechanics. We start with the elastic wave equation for the scalar displacement field $U(\mathbf{X})$ of shear waves written in the harmonic regime

$$
\operatorname{div}(\mu \nabla U)+\rho \omega^{2} U=0
$$

\footnotetext{
*Laboratoire de Mécanique des Solides, Route de Saclay, 91128 Palaiseau, France, (marigo@lms.polytechnique.fr)

†Institut Langevin, 1 rue Jussieu, 75005 Paris, France (agnes.maurel@espci.fr)
} 


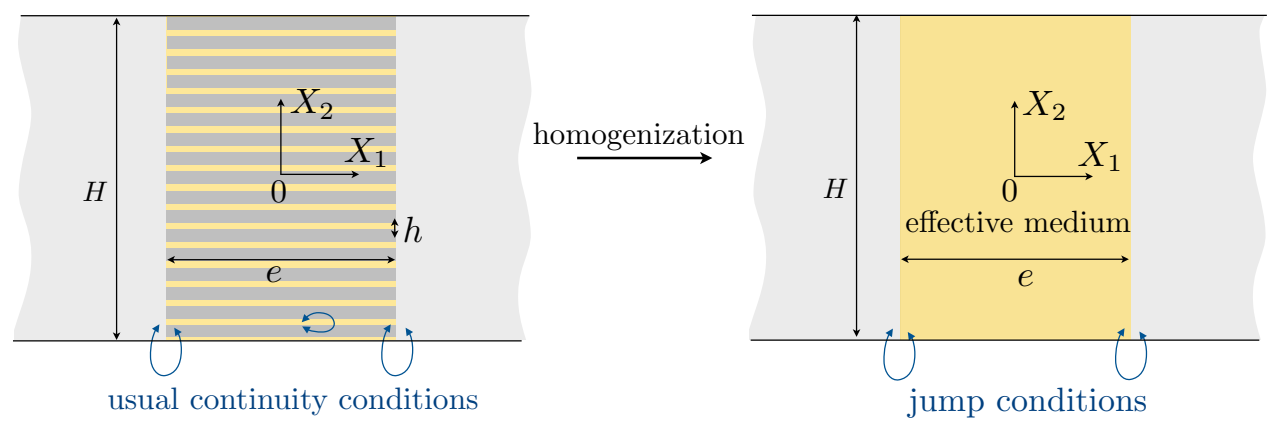

FIGURE 1. On the left, the wave problem is set for a slab filled with a stratified medium, with the usual continuity conditions on the boundaries of the layers. On the right, it is set for an equivalent slab made of an effective medium (homogeneous and anisotropic) and jump conditions apply at the boundaries of the slab $X_{1}= \pm e / 2$ (the boundaries at $\left|X_{2}\right|=H / 2$ are disregarded or equivalently, we consider $H \rightarrow+\infty)$.

with $\mu(\mathbf{X})$ the shear modulus and $\rho(\mathbf{X})$ the mass density being spatially dependent of $\mathbf{X}=\left(X_{1}, X_{2}\right) \in \mathbb{R} \times(-H / 2, H / 2)$ and $\omega$ the frequency (Fig. 1). Eq. (1) can be written using the non dimensional parameters

$$
\alpha(\mathbf{X}) \equiv \frac{\mu(\mathbf{X})}{\mu_{\mathrm{m}}}, \quad \text { and } \quad \beta(\mathbf{X}) \equiv \frac{\rho(\mathbf{X})}{\rho_{\mathrm{m}}},
$$

with $\left(\mu_{\mathrm{m}}, \rho_{\mathrm{m}}\right)$ the shear modulus and mass density of the medium surrounding the stratified structure, hereafter called the substrate; with $k=\omega \sqrt{\rho_{\mathrm{m}} / \mu_{\mathrm{m}}}$ the wavenumber in the substrate, we get

$$
\operatorname{div}(\alpha \nabla U)+\beta k^{2} U=0,
$$

which also applies to acoustic waves, to transverse magnetic or transverse electric polarized electromagnetic waves or to scalar (shear) elastic waves. It follows that the Helmholtz equation $\Delta U+k^{2} U=0$ applies in the substrate by construction. We shall establish that the homogenized wave equation, up to second order, is of the form

$$
\operatorname{div} \boldsymbol{\Sigma}+\beta^{\text {eff }} k^{2} U=0, \quad \boldsymbol{\Sigma}=\left(\begin{array}{cc}
\alpha_{1}^{\text {eff }} & 0 \\
0 & \alpha_{2}^{\text {eff }}
\end{array}\right) \nabla U,
$$

where $\left(\alpha_{1}^{\text {eff }}, \alpha_{2}^{\text {eff }}, \beta^{\text {eff }}\right)$ will be defined in subsection 2.2 , see (27). Next, for the stratified medium occupying the region $\mathbf{X}=\left(X_{1}, X_{2}\right) \in(-e / 2, e / 2) \times(-H / 2, H / 2)$, the homogenized slab, in which (3) applies, occupies the same region and effective continuity or discontinuity conditions apply at $X_{1}= \pm e / 2$. While the usual continuities of the displacement $U$ and of the normal stress $\boldsymbol{\Sigma} \cdot \mathbf{N}$ are obtained at the first order, discontinuities of these quantities at second order are established. Specifically, the jump conditions read

$$
\left\{\begin{array}{l}
\llbracket U \rrbracket=\frac{h \mathcal{B}}{2}\left(\boldsymbol{\Sigma}^{-}+\boldsymbol{\Sigma}^{+}\right) \cdot \mathbf{N}, \\
\llbracket \boldsymbol{\Sigma} \rrbracket \cdot \mathbf{N}=-\frac{h \mathcal{C}}{2}\left(\frac{\partial^{2} U^{-}}{\partial X_{2}^{2}}+\frac{\partial^{2} U^{+}}{\partial X_{2}^{2}}\right) .
\end{array}\right.
$$

In the above relations, for any field $V$ being discontinuous across a boundary with $\left(V^{-}, V^{+}\right)$its values on both sides, we have defined the jump $\llbracket V \rrbracket \equiv V^{+}-V^{-}$(and 

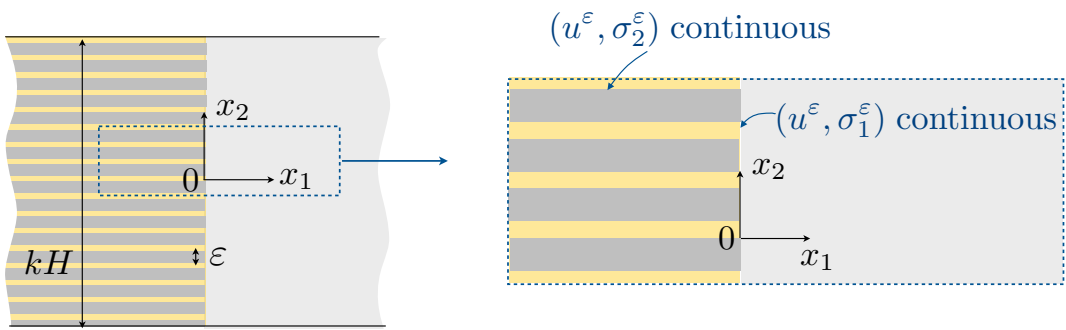

FIGURE 2. Single interface between the stratified medium occupying the region $x_{1}<0$ and the substrate occupying the region $x_{1}>0$. The usual continuity conditions apply at the boundaries between the layers $\left(u^{\varepsilon}, \sigma_{2}^{\varepsilon}\right)$ and at the boundaries between the layers and the substrate $\left(u^{\varepsilon}, \sigma_{1}^{\varepsilon}\right)$ at $x_{1}=0$.

the convention \pm refers to the direction of the normal $\mathbf{N})$. The parameters $(\mathcal{B}, \mathcal{C})$ entering in the jump conditions are deduced from elementary problems, being the equivalent of the cell problems in the classical homogenization, Eqs. (34) and (35). In the absence of high contrasts resulting in possible resonances in one or several layers (and possible resonances are disregarded here), these problems are static problems that can be solved once and for all. Finally, the problem of the boundary layers at the boundaries $X_{2}= \pm H / 2$ would require a specific treatment and they are disregarded in the present paper (alternatively, we may consider $H \rightarrow+\infty$ ).

Validations of our homogenization method are presented in section 3 by comparison with full wave numerics. We restrict ourselves to the case of layers associated with Neumann boundary conditions ; it corresponds to cracks or voids in elasticity, to sound hard inclusions in acoustics or to perfectly conducting metals in electromagnetism. This case allows for explicit expressions of the interface parameters $(\mathcal{B}, \mathcal{C})$, Eq. (53) (see also subsection S2.1). The limitations of the present approach to small slab thicknesses are discussed in Appendix A. Finally, we report in section S1 the case of a stratified slab with one boundary being free of stresses (associated with Neumann boundary condition) and details on the numerical resolutions are given in section S2.

2. Up to second-order homogenization. In this section, we shall work on a problem simplified with respect to the one in Fig. 1 in the sense that we consider a single interface (also, we shall work in dimensionless coordinates). We define $x_{1}=$ $k\left(X_{1}-e / 2\right), x_{2}=k X_{2}$, which means that we focus on a region near the boundary of the stratified medium at $X_{1}=e / 2$ in the original problem in Fig. 1(a). But now, we assume that the stratified medium occupies the region $x_{1}<0$, Fig. 2. Doing so, we assumed implicitly that the wave passing through the stratified slab in the configuration of the Fig. 1 feels the boundaries and the bulk of the stratified medium. To anticipate, this means that the slab is thick enough, and thick means that the evanescent fields at both boundaries of the slab do not interact. If it is not the case, one should consider the whole thin slab in the asymptotic analysis, as done in $[5,6,9,11]$ (this is discussed further in Appendix A).

We shall define the actual problem for $\mathbf{x}=\left(x_{1}, x_{2}\right) \in \mathbb{R} \times(-k H / 2, k H / 2)$. With the periodicity of the stratified medium $\varepsilon=k h \ll 1$, the solution of the problem depends on $\varepsilon$ and we make this dependence to appear explicitly, by denoting $a^{\varepsilon}(\mathbf{x}) \equiv$ $\alpha(\mathbf{X}), b^{\varepsilon}(\mathbf{x}) \equiv \beta(\mathbf{X})$ and $u^{\varepsilon}(\mathbf{x}) \equiv U(\mathbf{X}), \boldsymbol{\sigma}^{\varepsilon}(\mathbf{x}) \equiv k^{-1} \alpha(\mathbf{X}) \boldsymbol{\nabla} U(\mathbf{X})$. In x-coordinate, 
(2) reads

$$
\begin{aligned}
& \operatorname{div} \boldsymbol{\sigma}^{\varepsilon}(\mathbf{x})+b^{\varepsilon}(\mathbf{x}) u^{\varepsilon}(\mathbf{x})=0 \\
& \boldsymbol{\sigma}^{\varepsilon}(\mathbf{x})=a^{\varepsilon}(\mathbf{x}) \boldsymbol{\nabla} u^{\varepsilon}(\mathbf{x})
\end{aligned}
$$

and

$$
a^{\varepsilon}(\mathbf{x})=\left\{\begin{array}{ll}
1, & x_{1}>0, \\
a\left(\frac{x_{2}}{\varepsilon}\right), & x_{1}<0 .
\end{array} \quad b^{\varepsilon}(\mathbf{x})= \begin{cases}1, & x_{1}>0 \\
b\left(\frac{x_{2}}{\varepsilon}\right), & x_{1}<0 .\end{cases}\right.
$$

The functions $a$ and $b$ are 1-periodic and piecewise constant; at this point, it is not necessary to define them more specifically. To (5), we have to associate boundary conditions. At each boundary between two different media, the continuity of the displacement $u^{\varepsilon}$ and of the normal stress $\boldsymbol{\sigma}^{\varepsilon} \cdot \mathbf{n}$ apply (with $\mathbf{n}$ the vector normal to the boundary); this applies at the boundaries between two layers within the stratified medium and at the boundaries between the layers and the substrate at $x_{1}=0$ (Fig. $2)$. Finally, once the wave source is defined, the conditions satisfied by $\left(u^{\varepsilon}, \boldsymbol{\sigma}^{\varepsilon}\right)$ for $x_{2}= \pm k H / 2$ and for $\left|x_{1}\right| \rightarrow+\infty$, often referred as to radiation conditions, can be defined; for the time being, we do not need to specify them.

2.1. The asymptotic analysis. The idea is to define three regions where different asymptotic expansions will be used, Eqs. (7). The inner region contains the boundary between the stratified medium and the substrate. Roughly speaking, it is the region where the boundary layer effects are significant; in terms of wavefield, this means that the inner region contains the so-called evanescent field vanishing far from the boundary. The two outer regions for $x_{1}>0$ and $x_{1}<0$ are the regions far enough from the interface, where the evanescent field can be neglected. Next, the inner region and the outer regions are connected using so -called matching conditions, which will constitute the boundary conditions for the outer solutions.
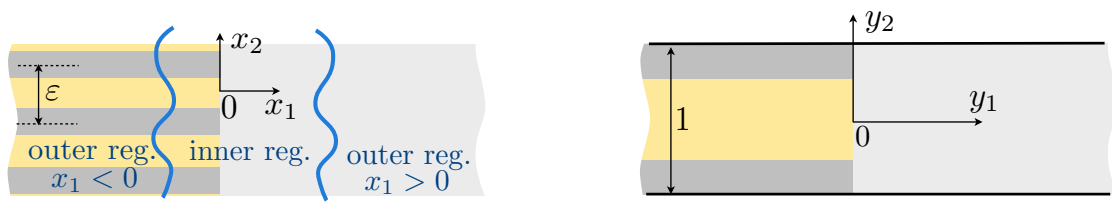

Figure 3. On the left, configuration in $\mathbf{x}$ coordinate; the periodicity along $x_{2}$ is $\varepsilon \equiv k h$; the inner region corresponds to the neighborhood of the boundary between the stratified medium $\left(x_{1}<0\right)$ and the substrate being a homogeneous medium $\left(x_{1}>0\right)$. On the right, the unit cell (inner region) in $\mathbf{y}$ coordinate, with $\mathbf{y}=\mathbf{x} / \varepsilon$, and $\mathbf{y} \in \mathbb{R} \times Y$, with $Y=(-1 / 2,1 / 2)$.

2.1.1. The outer and inner expansions. As in the classical homogenization, the asymptotic expansions are thought with spatial dependences on a macroscopic coordinate $\mathbf{x}$ associated with slow variations of the fields (with the typical scale $1 / k$ of the wave) and a microscopic coordinate $\mathbf{y}$, associated with rapid variations (the typical scale $h$ of the layers), and in each region, we keep the coordinates that are relevant to describe the variations of the field. To do so, we define $\mathbf{y} \equiv \mathbf{x} / \varepsilon$ and we 
assume that $\left(u^{\varepsilon}, \boldsymbol{\sigma}^{\varepsilon}\right)$ can be expanded by using the following asymptotic expansions

$$
\begin{cases}\text { outer region } x_{1}>0, & u^{\varepsilon}=u^{0}(\mathbf{x})+\varepsilon u^{1}(\mathbf{x})+\ldots, \\ & \boldsymbol{\sigma}^{\varepsilon}=\boldsymbol{\sigma}^{0}(\mathbf{x})+\varepsilon \boldsymbol{\sigma}^{1}(\mathbf{x})+\ldots, \\ \text { outer region } x_{1}<0, & u^{\varepsilon}=u^{0}\left(\mathbf{x}, y_{2}\right)+\varepsilon u^{1}\left(\mathbf{x}, y_{2}\right)+\ldots, \\ & \boldsymbol{\sigma}^{\varepsilon}=\boldsymbol{\sigma}^{0}\left(\mathbf{x}, y_{2}\right)+\varepsilon \boldsymbol{\sigma}^{1}\left(\mathbf{x}, y_{2}\right)+\ldots, \\ \text { inner region, } & u^{\varepsilon}=v^{0}\left(x_{2}, \mathbf{y}\right)+\varepsilon v^{1}\left(x_{2}, \mathbf{y}\right)+\ldots \\ & \boldsymbol{\sigma}^{\varepsilon}=\boldsymbol{\tau}^{0}\left(x_{2}, \mathbf{y}\right)+\varepsilon \boldsymbol{\tau}^{1}\left(x_{2}, \mathbf{y}\right)+\ldots\end{cases}
$$

with the outer terms $\left(u^{n}, \boldsymbol{\sigma}^{n}\right)$ for $x_{1}<0$ and the inner terms $\left(v^{n}, \boldsymbol{\tau}^{n}\right)$ being $Y$-periodic with $Y=(-1 / 2,1 / 2)$. Thus, we shall consider $y_{2} \in Y$ and in the inner region $y_{1} \in \mathbb{R}$; next, in the three regions $x_{2} \in(-k H / 2, k H / 2)$ and in the outer regions $x_{1} \in(-\infty, 0)$ and $x_{1} \in(0,+\infty)$ respectively.

The differential operator reads, in the different regions, as

$$
\left\{\begin{array}{cll}
\text { in the outer region, } & \boldsymbol{\nabla} \rightarrow \boldsymbol{\nabla}_{\mathbf{x}}, & x_{1}>0, \\
\boldsymbol{\nabla} & \rightarrow \boldsymbol{\nabla}_{\mathbf{x}}+\frac{1}{\varepsilon} \frac{\partial}{\partial y_{2}} \mathbf{e}_{2}, & x_{1}<0, \\
\text { in the inner region, } & \boldsymbol{\nabla} \rightarrow \frac{\partial}{\partial x_{2}} \mathbf{e}_{2}+\frac{1}{\varepsilon} \boldsymbol{\nabla}_{\mathbf{y}}, &
\end{array}\right.
$$

where $\boldsymbol{\nabla}_{\mathbf{x}}$ means gradient w.r.t. $\mathbf{x}$ and $\boldsymbol{\nabla}_{\mathbf{y}}$ means gradient w.r.t. $\mathbf{y}$. Let us comment the dependences in $\mathbf{x}$ and $\mathbf{y}$ of the fields in each region, (7). The inner solution is characterized by rapid variations of the evanescent field, and these variations are naturally described by $\mathbf{y}\left(|\boldsymbol{\nabla} U| \sim U / h\right.$ gives $\left.\left|\nabla_{\mathbf{y}} u\right| \sim u\right)$. But the inner solution contains also the propagating field associated with slow variations along $x_{2}$, typically the phase variation along the boundary at $x_{1}=0$; this is taken into account by keeping $x_{2}$ as an additional coordinate. In the outer region $x_{1}>0$, there is no rapid variations due to the evanescent field (this latter being confined in the inner region, by definition); thus, we need only the coordinates $\mathbf{x}$ which describe the propagating field with $|\nabla U| \sim k U$ and thus $\nabla_{\mathbf{x}} u \sim u$. The story is different for $x_{1}<0$; there, the field has still slow variations, but it also experiences rapid variations across the layers and this is accounted for by keeping the coordinate $y_{2}$.

Finally, from $(6),\left(a^{\varepsilon}, b^{\varepsilon}\right)$ can be specified with in the outer regions

$$
\left\{\begin{array}{lll}
\text { outer region } x_{1}>0, & a^{\varepsilon}(\mathbf{x})=1, & b^{\varepsilon}(\mathbf{x})=1, \\
\text { outer region } x_{1}<0, & a^{\varepsilon}(\mathbf{x})=a\left(\frac{x_{2}}{\varepsilon}\right), & b^{\varepsilon}(\mathbf{x})=b\left(\frac{x_{2}}{\varepsilon}\right),
\end{array}\right.
$$

and in the inner region $a^{\varepsilon}(\mathbf{x})=\tilde{a}(\mathbf{x} / \varepsilon)$ and $b^{\varepsilon}(\mathbf{x})=\tilde{b}(\mathbf{x} / \varepsilon)$ with

$$
\tilde{a}(\mathbf{y})=\left\{\begin{array}{ll}
a\left(y_{2}\right), & y_{1}<0, \\
1, & y_{1}>0,
\end{array} \quad \tilde{b}(\mathbf{y})= \begin{cases}b\left(y_{2}\right), & y_{1}<0 \\
1, & y_{1}>0\end{cases}\right.
$$

with $a\left(y_{2}\right), b\left(y_{2}\right) 1$-periodic and piecewise constant. 
2.1.2. The boundary conditions and the matching conditions. The inner and outer problems have to be associated with boundary conditions or radiation conditions which ensure that the problems are well-posed. For the inner solution, the continuities of the displacement and of the normal stress apply at the boundaries between two layers within the stratified medium and at the boundaries between the layers and the substrate at $y_{1}=0$, whence

$$
v^{n}, \boldsymbol{\tau}^{n} \cdot \mathbf{n} \text { are continuous everywhere, } n=0,1 \ldots,
$$

but the conditions at infinity are unknown a priori. Reversely, since the outer expansions hold true only far away from the interface, the outer terms satisfy the radiation condition (once defined) but they do not have to satisfy the continuity conditions at $x_{1}=0$; only the conditions of continuity of $u^{n}$ and $\boldsymbol{\sigma}^{n} \cdot \mathbf{n}$ at the boundaries between the layers within the stratified medium apply for $x_{1}<0$ (thus, with $\mathbf{n}=\mathbf{e}_{2}$ ).

The missing conditions for the inner and outer terms are provided simultaneously by the matching conditions (see the discussion on alternative matching in [1]), at leading order

$$
\begin{aligned}
& u^{0}\left(0^{-}, x_{2}, y_{2}\right)=\lim _{y_{1} \rightarrow-\infty} v^{0}\left(x_{2}, \mathbf{y}\right), \\
& u^{0}\left(0^{+}, x_{2}\right)=\lim _{y_{1} \rightarrow+\infty} v^{0}\left(x_{2}, \mathbf{y}\right), \\
& \boldsymbol{\sigma}^{0}\left(0^{-}, x_{2}, y_{2}\right)=\lim _{y_{1} \rightarrow-\infty} \boldsymbol{\tau}^{0}\left(x_{2}, \mathbf{y}\right), \\
& \boldsymbol{\sigma}^{0}\left(0^{+}, x_{2}\right)=\lim _{y_{1} \rightarrow+\infty} \boldsymbol{\tau}^{0}\left(x_{2}, \mathbf{y}\right),
\end{aligned}
$$

and at order $\varepsilon$

$$
\begin{aligned}
& u^{1}\left(0^{-}, x_{2}, y_{2}\right)=\lim _{y_{1} \rightarrow-\infty}\left[v^{1}\left(x_{2}, \mathbf{y}\right)-y_{1} \frac{\partial u^{0}}{\partial x_{1}}\left(0^{-}, x_{2}, y_{2}\right)\right], \\
& u^{1}\left(0^{+}, x_{2}\right)=\lim _{y_{1} \rightarrow+\infty}\left[v^{1}\left(x_{2}, \mathbf{y}\right)-y_{1} \frac{\partial u^{0}}{\partial x_{1}}\left(0^{+}, x_{2}\right)\right], \\
& \boldsymbol{\sigma}^{1}\left(0^{-}, x_{2}, y_{2}\right)=\lim _{y_{1} \rightarrow-\infty}\left[\boldsymbol{\tau}^{1}\left(x_{2}, \mathbf{y}\right)-y_{1} \frac{\partial \boldsymbol{\sigma}^{0}}{\partial x_{1}}\left(0^{-}, x_{2}, y_{2}\right)\right], \\
& \boldsymbol{\sigma}^{1}\left(0^{+}, x_{2}\right)=\lim _{y_{1} \rightarrow+\infty}\left[\boldsymbol{\tau}^{1}\left(x_{2}, \mathbf{y}\right)-y_{1} \frac{\partial \boldsymbol{\sigma}^{0}}{\partial x_{1}}\left(0^{+}, x_{2}\right)\right] .
\end{aligned}
$$

At order $\varepsilon$, the conditions are obtained using the Taylor expansions of $\left(u^{0}, \boldsymbol{\sigma}^{0}\right)$, for instance for $x_{1}>0, u^{0}(\mathbf{x})=u^{0}\left(0^{+}, x_{2}\right)+x_{1} \partial_{x_{1}} u^{0}\left(0^{+}, x_{2}\right)+\cdots=u^{0}\left(0^{+}, x_{2}\right)+$ $\varepsilon y_{1} \partial_{x_{1}} u^{0}\left(0^{+}, x_{2}\right)+\ldots$

2.2. The homogenized wave equation. We want to establish the wave equation, up to second-order, satisfied by the mean fields $(\bar{u}(\mathbf{x}), \overline{\boldsymbol{\sigma}}(\mathbf{x}))$ with

$$
\bar{u} \equiv\left\langle u^{0}\right\rangle+\varepsilon\left\langle u^{1}\right\rangle, \quad \overline{\boldsymbol{\sigma}} \equiv\left\langle\boldsymbol{\sigma}^{0}\right\rangle+\varepsilon\left\langle\boldsymbol{\sigma}^{1}\right\rangle .
$$

We have defined the average over $y_{2} \in Y$ for any function $f$

$$
\langle f\rangle(\mathbf{x}) \equiv \int_{Y} \mathrm{~d} y_{2} f\left(\mathbf{x}, y_{2}\right),
$$

and obviously, if $f$ does not depend on $y_{2},\langle f\rangle=f$. 
The homogenized wave equation is sought for $x_{1}<0$ only. For $x_{1}>0$, from (5) along with (9), the wave equation is

$$
\operatorname{div}_{\mathbf{x}} \boldsymbol{\sigma}^{n}+u^{n}=0, \quad \boldsymbol{\sigma}^{n}=\nabla_{\mathbf{x}} u^{n}, \quad(n=0,1), \quad \text { for } x_{1}>0,
$$

being the same at each order, and the fields being independent of $y_{2}$ equal their averages.

2.2.1. The homogenized wave equation in $x_{1}<0$ at first order. For $x_{1}<0$, the Eqs. (5), at leading order $(1 / \varepsilon)$, read $\partial_{y_{2}} \sigma_{2}^{0}=0=\partial_{y_{2}} u^{0}$, whence we can note

$$
u^{0}\left(\mathbf{x}, y_{2}\right)=u^{0}(\mathbf{x}), \quad \sigma_{2}^{0}\left(\mathbf{x}, y_{2}\right)=\sigma_{2}^{0}(\mathbf{x})
$$

and $u^{0}, \sigma_{2}^{0}$ equal their averages. Now, we establish the relation between $\left\langle\boldsymbol{\sigma}^{0}\right\rangle$ and $u^{0}$. The Eqs. (5) at order $\varepsilon^{0}$ in the outer problems $x_{1}<0$ give

$$
\boldsymbol{\sigma}^{0}\left(\mathbf{x}, y_{2}\right)=a\left(y_{2}\right)\left[\boldsymbol{\nabla}_{\mathbf{x}} u^{0}(\mathbf{x})+\frac{\partial u^{1}}{\partial y_{2}}\left(\mathbf{x}, y_{2}\right) \mathbf{e}_{2}\right],
$$

and

$$
\operatorname{div}_{\mathbf{x}} \boldsymbol{\sigma}^{0}\left(\mathbf{x}, y_{2}\right)+\frac{\partial \sigma_{2}^{1}}{\partial y_{2}}\left(\mathbf{x}, y_{2}\right)+b\left(y_{2}\right) u^{0}(\mathbf{x})=0
$$

Averaging both equations, with $\boldsymbol{\sigma}^{0}\left(\mathbf{x}, y_{2}\right)=\sigma_{1}^{0}\left(\mathbf{x}, y_{2}\right) \mathbf{e}_{1}+\sigma_{2}^{0}(\mathbf{x}) \mathbf{e}_{2}$, and owing to the periodicity of $u^{1}$ and of $\sigma_{2}^{1}$ w.r.t. $y_{2}$ (thus, $\left\langle\partial_{y_{2}} u^{1}\right\rangle=0=\left\langle\partial_{y_{2}} \sigma_{2}^{1}\right\rangle$ ), we easily get the wave equation at the first order

$$
\begin{aligned}
& \left\langle\boldsymbol{\sigma}^{0}\right\rangle(\mathbf{x})=\langle a\rangle \frac{\partial u^{0}}{\partial x_{1}}(\mathbf{x}) \mathbf{e}_{1}+\langle 1 / a\rangle^{-1} \frac{\partial u^{0}}{\partial x_{2}}(\mathbf{x}) \mathbf{e}_{2}, \\
& \operatorname{div}_{\mathbf{x}}\left\langle\boldsymbol{\sigma}^{0}\right\rangle+\langle b\rangle u^{0}=0 .
\end{aligned}
$$

2.2.2. Second-order - useful relations. Before going to the next order, we shall establish the relations (22) for $u^{1}$ and (24) for $\sigma_{2}^{1}$, that we shall use later. Both relations use the same property : consider a piecewise differentiable function $g(y)$, with $g^{\prime}(y)$ even; then $(g-\langle g\rangle)$ is odd and, for any function $f(y)$ being even, $f(g-\langle g\rangle)$ is odd. Thus, the integral over $y \in Y$ vanishes, from which $\langle f g\rangle=\langle f\rangle\langle g\rangle$.

First, from (18), we have

$$
\frac{\partial u^{1}}{\partial y_{2}}\left(\mathbf{x}, y_{2}\right)=\frac{1 / a\left(y_{2}\right)-\langle 1 / a\rangle}{\langle 1 / a\rangle} \frac{\partial u^{0}}{\partial x_{2}}(\mathbf{x})
$$

and we used that $\sigma_{2}^{0}(\mathbf{x})=\langle 1 / a\rangle^{-1} \partial_{x_{2}} u^{0}(\mathbf{x})$ from (20a). Because $a\left(y_{2}\right)$ is even, $\partial_{y_{2}} u^{1}$ is even too, so the property on the average applies and

$$
\left\langle f(\cdot) u^{1}(\mathbf{x}, \cdot)\right\rangle=\langle f\rangle\left\langle u^{1}\right\rangle(\mathbf{x}), \quad \text { for any even } f .
$$

Integrating (21), we also have

$$
u^{1}\left(\mathbf{x}, y_{2}\right)=A\left(y_{2}\right) \frac{\partial u^{0}}{\partial x_{2}}(\mathbf{x})+\left\langle u^{1}\right\rangle(\mathbf{x}), \quad A\left(y_{2}\right) \equiv \int_{-1 / 2}^{y_{2}} \mathrm{~d} y \frac{1 / a(y)-\langle 1 / a\rangle}{\langle 1 / a\rangle} .
$$

In the above expression, we have used that $\langle A\rangle=0$, by construction. 
Next, we use that $\sigma_{1}^{0}\left(\mathbf{x}, y_{2}\right)=a\left(y_{2}\right) \partial_{x_{1}} u^{0}(\mathbf{x})$ from (18), and thus $\left\langle\sigma_{1}^{0}\right\rangle(\mathbf{x})=$ $\langle a\rangle \partial_{x_{1}} u^{0}(\mathbf{x})$. Inserting the resulting relation $\sigma_{1}^{0}\left(\mathbf{x}, y_{2}\right)=a\left(y_{2}\right) /\langle a\rangle\left\langle\sigma_{1}^{0}\right\rangle(\mathbf{x})$ in (19), we get

$$
\frac{\partial \sigma_{2}^{1}}{\partial y_{2}}\left(\mathbf{x}, y_{2}\right)=-\frac{a\left(y_{2}\right)}{\langle a\rangle} \frac{\partial\left\langle\sigma_{1}^{0}\right\rangle}{\partial x_{1}}(\mathbf{x})-\frac{\partial \sigma_{2}^{0}}{\partial x_{2}}(\mathbf{x})-b\left(y_{2}\right) u^{0}(\mathbf{x})
$$

$(a, b)$ being even, $\partial_{y_{2}} \sigma_{2}^{1}$ is even w.r.t. $y_{2}$, from which the property on the average applies and

$$
\left\langle f(\cdot) \sigma_{2}^{1}(\mathbf{x}, \cdot)\right\rangle=\langle f\rangle\left\langle\sigma_{2}^{1}\right\rangle(\mathbf{x}), \text { for any even } f .
$$

2.2.3. The homogenized wave equation in $x_{1}<0$ at second-order. Now, we shall establish the relation between $\left\langle u^{1}\right\rangle$ and $\left\langle\boldsymbol{\sigma}^{1}\right\rangle$ (for $x_{1}<0$ ). Eq. (5b) at order $\varepsilon$ reads

$$
\boldsymbol{\sigma}^{1}\left(\mathbf{x}, y_{2}\right)=a\left(y_{2}\right)\left[\nabla_{\mathbf{x}} u^{1}\left(\mathbf{x}, y_{2}\right)+\frac{\partial u^{2}}{\partial y_{2}}\left(\mathbf{x}, y_{2}\right) \mathbf{e}_{2}\right] .
$$

To average the above equation, it is sufficient to use (22) and (24), with $a\left(y_{2}\right)$ being even. We get

$$
\begin{cases}\sigma_{1}^{1}\left(\mathbf{x}, y_{2}\right)=a\left(y_{2}\right) \frac{\partial u^{1}}{\partial x_{1}}\left(\mathbf{x}, y_{2}\right) & \rightarrow\left\langle\sigma_{1}^{1}\right\rangle(\mathbf{x})=\langle a\rangle \frac{\partial\left\langle u^{1}\right\rangle}{\partial x_{1}}(\mathbf{x}) \\ \frac{1}{a\left(y_{2}\right)} \sigma_{2}^{1}\left(\mathbf{x}, y_{2}\right)=\frac{\partial u^{1}}{\partial x_{2}}\left(\mathbf{x}, y_{2}\right)+\frac{\partial u^{2}}{\partial y_{2}}\left(\mathbf{x}, y_{2}\right) & \rightarrow\langle 1 / a\rangle\left\langle\sigma_{2}^{1}\right\rangle(\mathbf{x})=\frac{\partial\left\langle u^{1}\right\rangle}{\partial x_{2}}(\mathbf{x})\end{cases}
$$

where the arrow indicates the average process. Next, (5a) at order $\varepsilon$ reads

$$
\operatorname{div}_{\mathbf{x}} \boldsymbol{\sigma}^{1}\left(\mathbf{x}, y_{2}\right)+\frac{\partial \sigma_{2}^{2}}{\partial y_{2}}\left(\mathbf{x}, y_{2}\right)+b\left(y_{2}\right) u^{1}\left(\mathbf{x}, y_{2}\right)=0,
$$

whose average leads to

$$
\operatorname{div}_{\mathbf{x}}\left\langle\boldsymbol{\sigma}^{1}\right\rangle(\mathbf{x})+\langle b\rangle\left\langle u^{1}\right\rangle(\mathbf{x})=0,
$$

and we have used $\left\langle b u^{1}\right\rangle=\langle b\rangle\left\langle u^{1}\right\rangle$ from (22) and $\left\langle\partial_{y_{2}} \sigma_{2}^{2}\right\rangle=0$ because $\sigma_{2}^{2}$ is periodic w.r.t. $y_{2}$.

2.2.4. Up to second-order homogenized wave equation. It is now sufficient to gather (20) and (25)-(26) to get the homogenized wave equation up to second order for $(\bar{u}(\mathbf{x}), \overline{\boldsymbol{\sigma}}(\mathbf{x}))$ in $(14)$

$$
\operatorname{div} \overline{\boldsymbol{\sigma}}+\langle b\rangle \bar{u}=0, \quad \overline{\boldsymbol{\sigma}}=\left(\begin{array}{cc}
\langle a\rangle & 0 \\
0 & \langle 1 / a\rangle^{-1}
\end{array}\right) \nabla \bar{u}, \quad \text { for } x_{1}<0,
$$

which coincides, when coming back to the real space, to (3).

2.3. Jump conditions. To the homogenized wave equation (27), we have to associate jump conditions at the interface $x_{1}=0$. In this section, we will show that the usual continuities of the displacement and of the normal stress are obtained at leading order, while the second order makes discontinuities of these two fields to appear. To that aim, we have to consider the inner solution and its matching with the two outer solutions. We are looking for the jumps of $\left(\bar{u}, \overline{\sigma_{1}}\right)$ defined in $(14)$,

$$
\llbracket \bar{u} \rrbracket=\llbracket u^{0} \rrbracket+\varepsilon \llbracket\left\langle u^{1}\right\rangle \rrbracket, \quad \llbracket \overline{\sigma_{1}} \rrbracket=\llbracket\left\langle\sigma_{1}^{0}\right\rangle \rrbracket+\varepsilon \llbracket\left\langle\sigma_{1}^{1}\right\rangle \rrbracket,
$$


and their expressions in terms of $\bar{u}^{ \pm}$and $\overline{\boldsymbol{\sigma}}^{ \pm}$, the values of $\bar{u}$ and $\overline{\boldsymbol{\sigma}}$ on both sides of the interface (at this stage, we have to distinguish the values on both sides, the fields being discontinuous).

2.3.1. The jump conditions at the first order. Eq. (5b) for the inner problem at the leading order in $1 / \varepsilon$ tells us that $\nabla_{\mathbf{y}} v^{0}=0$ from which $v^{0}$ does not depend on $\mathbf{y}$. From the previous section, we already know that $u^{0}(\mathbf{x})$ does not depend on $y_{2}$, from which the matching conditions, (12a) and (12b), give

$$
u^{0}\left(0^{-}, x_{2}\right)=u^{0}\left(0^{+}, x_{2}\right)=v^{0}\left(x_{2}\right), \quad \text { and } \llbracket u^{0} \rrbracket=0 .
$$

Next, (5a) in the inner region gives at the leading order $\operatorname{div}_{\mathbf{y}} \tau^{0}=0$; by integrating this equation on $\mathbb{R} \times Y$, we get

$$
\int_{Y} \mathrm{~d} y_{2}\left[\tau_{1}^{0}\left(x_{2},+\infty, y_{2}\right)-\tau_{1}^{0}\left(x_{2},-\infty, y_{2}\right)\right]=0,
$$

(we have used the periodicity of $\boldsymbol{\tau}^{0}$ w.r.t. $y_{2}$ and the continuity of $\boldsymbol{\tau}^{0} \cdot \mathbf{n}$ between the layers along $y_{2}$ ). From the matching conditions (12c)-(12d) integrated over $Y$, we get

$$
\left\langle\sigma_{1}^{0}\right\rangle\left(0^{-}, x_{2}\right)=\sigma_{1}^{0}\left(0^{+}, x_{2}\right), \text { and } \llbracket\left\langle\sigma_{1}^{0}\right\rangle \rrbracket=0 .
$$

At first order, the usual continuities of the displacement and of the normal stress are obtained. To capture the effect of the boundary layers in the neighborhood of $x_{1}=0$, we have to go up to the second order.

2.3.2. The elementary problems. Before going to the second order, we need to inspect further the inner solution. There, the variations of $\tilde{a}(\mathbf{y})$ and $\tilde{b}(\mathbf{y})$ in (5) are more involved than the simple forms $a\left(y_{2}\right)$ and $b\left(y_{2}\right)$ considered until now for $x_{1}<0$, see (10). The difference between $\tilde{a}(\mathbf{y})$ and $a\left(y_{2}\right)$ will constitute the whole story. From (5a) at order $\varepsilon^{-1}$ and (5b) at order $\varepsilon^{0}$, we have

$$
\operatorname{div}_{\mathbf{y}} \tau^{0}=0, \quad \tau^{0}\left(x_{2}, \mathbf{y}\right)=\tilde{a}(\mathbf{y})\left[\frac{\partial u^{0}}{\partial x_{2}}\left(0, x_{2}\right) \mathbf{e}_{2}+\nabla_{\mathbf{y}} v^{1}\left(x_{2}, \mathbf{y}\right)\right],
$$

where we used that $\partial_{x_{2}} u^{0}(\mathbf{x})$ is continuous at $x_{1}=0$ as $u^{0}$ does from (29). The matching conditions $(12 \mathrm{c})-(12 \mathrm{~d})$ yield

$$
\left\{\begin{aligned}
\boldsymbol{\tau}^{0}\left(x_{2},-\infty, y_{2}\right) & =\frac{a\left(y_{2}\right)}{\langle a\rangle}\left\langle\sigma_{1}^{0}\right\rangle\left(0, x_{2}\right) \mathbf{e}_{1}+\langle 1 / a\rangle^{-1} \frac{\partial u^{0}}{\partial x_{2}}\left(0, x_{2}\right) \mathbf{e}_{2} \\
& =a\left(y_{2}\right) \frac{\partial u^{0}}{\partial x_{2}}\left(0, x_{2}\right) \mathbf{e}_{2}+a\left(y_{2}\right) \nabla_{\mathbf{y}} v^{1}\left(x_{2},-\infty, y_{2}\right) . \\
\boldsymbol{\tau}^{0}\left(x_{2},+\infty, y_{2}\right) & =\left\langle\sigma_{1}^{0}\right\rangle\left(0, x_{2}\right) \mathbf{e}_{1}+\frac{\partial u^{0}}{\partial x_{2}}\left(0, x_{2}\right) \mathbf{e}_{2} \\
& =\frac{\partial u^{0}}{\partial x_{2}}\left(0, x_{2}\right) \mathbf{e}_{2}+\nabla_{\mathbf{y}} v^{1}\left(x_{2},+\infty, y_{2}\right) .
\end{aligned}\right.
$$

For both limits above, the first line is given by the matching conditions with $\sigma^{0}$ expressed as a function of $\left\langle\sigma_{1}^{0}\right\rangle$ and $\partial_{x_{2}} u^{0}$; it is obvious for $x_{1}>0$, and for $x_{1}<0$, we used (18) and (20a) (with $\sigma_{2}^{0}=\left\langle\sigma_{2}^{0}\right\rangle$ from (17)). The second line is given by the expression of $\boldsymbol{\tau}^{0}$ in (31) along with (10). It follows that the system satisfied by 
$278 v^{1}\left(x_{2}, \mathbf{y}\right)$ can be written

(32)

$$
\left\{\begin{array}{l}
\operatorname{div}_{\mathbf{y}} \boldsymbol{\tau}^{0}=0, \text { with } \boldsymbol{\tau}^{0}=\tilde{a}(\mathbf{y})\left[\frac{\partial u^{0}}{\partial x_{2}}\left(0, x_{2}\right) \mathbf{e}_{2}+\nabla_{\mathbf{y}} v^{1}\left(x_{2}, \mathbf{y}\right)\right] \\
v^{1} \text { and } \boldsymbol{\tau}^{0} \cdot \mathbf{n} \text { continuous, } \\
\lim _{y_{1} \rightarrow-\infty} \nabla_{\mathbf{y}} v^{1}\left(x_{2}, \mathbf{y}\right)=\langle a\rangle^{-1}\left\langle\sigma_{1}^{0}\right\rangle\left(0, x_{2}\right) \mathbf{e}_{1}+\frac{1 / a\left(y_{2}\right)-\langle 1 / a\rangle}{\langle 1 / a\rangle} \frac{\partial u^{0}}{\partial x_{2}}\left(0, x_{2}\right) \mathbf{e}_{2}, \\
\lim _{y_{1} \rightarrow+\infty} \nabla_{\mathbf{y}} v^{1}\left(x_{2}, \mathbf{y}\right)=\left\langle\sigma_{1}^{0}\right\rangle\left(0, x_{2}\right) \mathbf{e}_{1},
\end{array}\right.
$$

with $v^{1}$ and $\boldsymbol{\tau}^{0}$ periodic w.r.t $y_{2}$, and we have used (11). The system (32) is linear w.r.t $\left\langle\sigma_{1}^{0}\right\rangle\left(0, x_{2}\right)$ and $\partial_{x_{2}} u^{0}\left(0, x_{2}\right)$. Thus, we define $V^{(1)}(\mathbf{y})$ and $V^{(2)}(\mathbf{y})$ such that

$$
\left\{\begin{array}{l}
v^{1}\left(x_{2}, \mathbf{y}\right)=\left\langle\sigma_{1}^{0}\right\rangle\left(0, x_{2}\right) V^{(1)}(\mathbf{y})+\frac{\partial u^{0}}{\partial x_{2}}\left(0, x_{2}\right)\left[A\left(y_{2}\right)+V^{(2)}(\mathbf{y})\right]+\hat{v}\left(x_{2}\right), \\
\boldsymbol{\tau}^{0}\left(x_{2}, \mathbf{y}\right)=\left\langle\sigma_{1}^{0}\right\rangle\left(0, x_{2}\right) \mathbf{T}^{(1)}(\mathbf{y})+\frac{\partial u^{0}}{\partial x_{2}}\left(0, x_{2}\right)\left[\frac{\tilde{a}(\mathbf{y}) / a\left(y_{2}\right)}{\langle 1 / a\rangle} \mathbf{e}_{2}+\mathbf{T}^{(2)}(\mathbf{y})\right],
\end{array}\right.
$$

with $\mathbf{T}^{(1)}(\mathbf{y}) \equiv \tilde{a}(\mathbf{y}) \boldsymbol{\nabla} V^{(1)}(\mathbf{y})$ and $\mathbf{T}^{(2)}(\mathbf{y}) \equiv \tilde{a}(\mathbf{y}) \boldsymbol{\nabla} V^{(2)}(\mathbf{y})$ (and $A\left(y_{2}\right)$ defined in $(23))$. Note that the field $v^{1}$ in (32) is defined up to a function of $x_{2}$, and it is denoted $\hat{v}\left(x_{2}\right)$ in (33); we shall see that the determination of $\hat{v}\left(x_{2}\right)$ is not needed. It is easy to see that if $\left(V^{(\mathrm{i})}, \mathbf{T}^{(\mathrm{i})}\right)$ satisfy the elementary problems

$$
\left\{\begin{array}{l}
\operatorname{div} \mathbf{T}^{(1)}=0, \quad \text { with } \mathbf{T}^{(1)}(\mathbf{y})=\tilde{a}(\mathbf{y}) \nabla V^{(1)}(\mathbf{y}) \\
V^{(1)} \text { and } \mathbf{T}^{(1)} \cdot \mathbf{n} \text { continuous, } \\
V^{(1)}, \mathbf{T}^{(1)} \text { periodic w.r.t. } y_{2} \\
\lim _{y_{1} \rightarrow-\infty} \nabla V^{(1)}(\mathbf{y})=\frac{\mathbf{e}_{1}}{\langle a\rangle}, \quad \lim _{y_{1} \rightarrow+\infty} \nabla V^{(1)}(\mathbf{y})=\mathbf{e}_{1},
\end{array}\right.
$$

and

$$
\left\{\begin{array}{l}
\operatorname{div}\left[\mathbf{T}^{(2)}+\frac{\tilde{a}(\mathbf{y}) / a\left(y_{2}\right)}{\langle 1 / a\rangle} \mathbf{e}_{2}\right]=0, \quad \text { with } \mathbf{T}^{(2)}(\mathbf{y})=\tilde{a}(\mathbf{y}) \nabla V^{(2)}(\mathbf{y}), \\
V^{(2)} \text { and }\left[\mathbf{T}^{(2)}+\frac{\tilde{a}(\mathbf{y}) / a\left(y_{2}\right)}{\langle 1 / a\rangle} \mathbf{e}_{2}\right] \cdot \mathbf{n} \text { continuous, } \\
V^{(2)}, \mathbf{T}^{(2)} \text { periodic } \text { w.r.t. } y_{2} \\
\lim _{y_{1} \rightarrow-\infty} \nabla V^{(2)}(\mathbf{y})=\mathbf{0}, \quad \lim _{y_{1} \rightarrow+\infty} \nabla V^{(2)}(\mathbf{y})=-\frac{1 / a\left(y_{2}\right)-\langle 1 / a\rangle}{\langle 1 / a\rangle} \mathbf{e}_{2},
\end{array}\right.
$$

then $v^{1}\left(x_{2}, \mathbf{y}\right)$ satisfies (32). The elementary solutions $V^{(1,2)}$ satisfy

$$
\left\{\begin{array} { l } 
{ \operatorname { l i m } _ { y _ { 1 } \rightarrow - \infty } [ V ^ { ( 1 ) } - \frac { y _ { 1 } } { \langle a \rangle } ] = - \mathcal { B } , } \\
{ \operatorname { l i m } _ { y _ { 1 } \rightarrow + \infty } [ V ^ { ( 1 ) } - y _ { 1 } ] = 0 , }
\end{array} \quad \left\{\begin{array}{l}
\lim _{y_{1} \rightarrow-\infty} V^{(2)}=-\mathcal{B}^{\prime} \\
\lim _{y_{1} \rightarrow+\infty} V^{(2)}=-A\left(y_{2}\right) .
\end{array}\right.\right.
$$

The above limits are obtained by integrating the limits of $\nabla V^{(\mathrm{i})}, i=1,2$, thus with unknown constants being a priori different at $y_{1} \rightarrow \pm \infty$. Next, because $V^{(\mathrm{i})}$ are defined 
in (34)-(35) up to a constant, we can set the constant equal to zero at $y_{1} \rightarrow+\infty$; for $V^{(1)}$, we denote $-\mathcal{B}$ the constant at $y_{1} \rightarrow-\infty$ (it is the first interface parameter). For $V^{(2)}$, it is denoted $-\mathcal{B}^{\prime}$; next, $V^{(2)}$ being odd w.r.t. $y_{2}$, we have $\mathcal{B}^{\prime}=0$. It is important to stress that the elementary problems, as the unit cell problems in the classical homogenization, can be solved once and for all, being written in the static limit. The relations between the elementary solutions $V^{(1,2)}$ and the evanescent fields in the actual problem (for a given scattering problem) are illustrated in Appendix A.

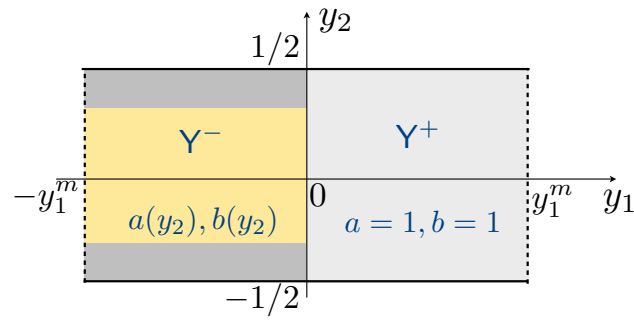

FIGURE 4. The domain $\mathbf{Y}=\mathbf{Y}^{-} \cup \mathbf{Y}^{+}$, with $\mathbf{Y}^{-}=\left(-y_{1}^{m}, 0\right) \times Y, \mathbf{Y}^{+}=\left(0,+y_{1}^{m}\right) \times Y . \tilde{a}(\mathbf{y})=a\left(y_{2}\right)$ and $\tilde{b}(\mathbf{y})=b\left(y_{2}\right)$ in $\mathbf{Y}^{-}$, and $a=1=b$ in $\mathbf{Y}^{+}$.

2.3.3. Jump conditions at second-order. Once the elementary problems are solved, it is possible to determine the jump conditions.

Jump of $\left\langle u^{1}\right\rangle$ - To get the jump of $\left\langle u^{1}\right\rangle$, it is sufficient to use the matching conditions (13a)-(13b) and we want $v^{1}\left(x_{2}, \pm \infty, y_{2}\right)$. From (33) along with (36), we have

$$
\left\{\begin{aligned}
v^{1}\left(x_{2},-\infty, y_{2}\right) & =\lim _{y_{1} \rightarrow-\infty}\left[\left(\frac{y_{1}}{\langle a\rangle}-\mathcal{B}\right)\left\langle\sigma_{1}^{0}\right\rangle\left(0, x_{2}\right)+A\left(y_{2}\right) \frac{\partial u^{0}}{\partial x_{2}}\left(0, x_{2}\right)+\hat{v}\left(x_{2}\right)\right] \\
v^{1}\left(x_{2},+\infty, y_{2}\right) & =\lim _{y_{1} \rightarrow+\infty}\left[y_{1}\left\langle\sigma_{1}^{0}\right\rangle\left(0, x_{2}\right)+\hat{v}\left(x_{2}\right)\right]
\end{aligned}\right.
$$

Now, we have $\left\langle\sigma_{1}^{0}\right\rangle\left(0, x_{2}\right)=\langle a\rangle \partial_{x_{1}} u^{0}\left(0^{-}, x_{2}\right)$ from (20a) and $\left\langle\sigma_{1}^{0}\right\rangle\left(0, x_{2}\right)=\partial_{x_{1}} u^{0}\left(0^{+}, x_{2}\right)$ from (16) and (30). Averaging $\llbracket u^{1} \rrbracket=u^{1}\left(0^{+}, x_{2}\right)-u^{1}\left(0^{-}, x_{2}, y_{2}\right)$ over $Y$ and owing to $\langle A\rangle=0$, we get

$$
\llbracket\left\langle u^{1}\right\rangle \rrbracket=\mathcal{B}\left\langle\sigma_{1}^{0}\right\rangle\left(0, x_{2}\right) .
$$

Jump of $\left\langle\sigma_{1}^{1}\right\rangle$-The derivation of the jump of $\sigma_{1}^{1}$ is more tricky, or at least longer. First, we define $\mathrm{Y} \equiv\left(-y_{1}^{m}, y_{1}^{m}\right) \times Y$ (Figure 4) and we shall use the matching conditions (13c)-(13d) integrated over $Y$ and written in terms of $y_{1}^{m}$

$$
\left\{\begin{aligned}
\left\langle\sigma_{1}^{1}\right\rangle\left(0^{-}, x_{2}\right) & =\lim _{y_{1}^{m} \rightarrow+\infty}\left[\left\langle\tau_{1}^{1}\right\rangle\left(x_{2},-y_{1}^{m}\right)+y_{1}^{m} \frac{\partial\left\langle\sigma_{1}^{0}\right\rangle}{\partial x_{1}}\left(0^{-}, x_{2}\right)\right], \\
\left\langle\sigma_{1}^{1}\right\rangle\left(0^{+}, x_{2}\right) & =\lim _{y_{1}^{m} \rightarrow+\infty}\left[\left\langle\tau_{1}^{1}\right\rangle\left(x_{2}, y_{1}^{m}\right)-y_{1}^{m} \frac{\partial\left\langle\sigma_{1}^{0}\right\rangle}{\partial x_{1}}\left(0^{+}, x_{2}\right)\right] .
\end{aligned}\right.
$$

Integrating over $Y$ the Eq. (5a) written at order $\varepsilon^{0}$ for the inner problem, we get

$$
\int_{\mathbf{Y}} \mathrm{d} \mathbf{y}\left[\operatorname{div}_{\mathbf{y}} \boldsymbol{\tau}^{1}\left(x_{2}, \mathbf{y}\right)+\frac{\partial \tau_{2}^{0}}{\partial x_{2}}\left(x_{2}, \mathbf{y}\right)+\tilde{b}(\mathbf{y}) u^{0}\left(0, x_{2}\right)\right]=0 .
$$


Two of the three integrals above are easily obtained, namely

$$
\left\{\begin{array}{l}
\int_{Y} \mathrm{~d} \mathbf{y} \operatorname{div}_{\mathbf{y}} \boldsymbol{\tau}^{1}\left(x_{2}, \mathbf{y}\right)=\left\langle\tau_{1}^{1}\right\rangle\left(x_{2}, y_{1}^{m}\right)-\left\langle\tau_{1}^{1}\right\rangle\left(x_{2},-y_{1}^{m}\right), \\
\int_{Y} \mathrm{~d} \mathbf{y} \tilde{b}(\mathbf{y}) u^{0}\left(0, x_{2}\right)=y_{1}^{m}[1+\langle b\rangle] u^{0}\left(0, x_{2}\right) .
\end{array}\right.
$$

We used, for the first integral, the continuity of $\boldsymbol{\tau}^{1} \cdot \mathbf{n}$ and the periodicity of $\boldsymbol{\tau}^{1}$ w.r.t. $y_{2}$. Note that the first integral of $(40)$ corresponds to the first term in $\llbracket\left\langle\sigma_{1}^{1}\right\rangle \rrbracket$, from (38). For the second integral, we used (10).

Now, let us consider the second integral in (39). First, from (33), we have

$$
\frac{\partial \tau_{2}^{0}}{\partial x_{2}}\left(x_{2}, \mathbf{y}\right)=\frac{\partial\left\langle\sigma_{1}^{0}\right\rangle}{\partial x_{2}}\left(0, x_{2}\right) T_{2}^{(1)}(\mathbf{y})+\frac{\partial^{2} u^{0}}{\partial x_{2}^{2}}\left(0, x_{2}\right)\left[\frac{\tilde{a}(\mathbf{y}) / a\left(y_{2}\right)}{\langle 1 / a\rangle}+T_{2}^{(2)}(\mathbf{y})\right]
$$

Next, defining $\mathrm{Y}^{-}=\left(-y_{1}^{m}, 0\right) \times Y, \mathrm{Y}^{+}=\left(0,+y_{1}^{m}\right) \times Y$, and using (10), we get

$$
\left\{\begin{array}{l}
\int_{\mathrm{Y}^{-}} \mathrm{d} \mathbf{y} \frac{\tilde{a}(\mathbf{y}) / a\left(y_{2}\right)}{\langle 1 / a\rangle} \frac{\partial^{2} u^{0}}{\partial x_{2}^{2}}\left(0, x_{2}\right)=y_{1}^{m}\langle 1 / a\rangle^{-1} \frac{\partial^{2} u^{0}}{\partial x_{2}^{2}}\left(0, x_{2}\right), \\
\int_{\mathbf{Y}^{+}} \mathrm{d} \mathbf{y} \frac{\tilde{a}(\mathbf{y}) / a\left(y_{2}\right)}{\langle 1 / a\rangle} \frac{\partial^{2} u^{0}}{\partial x_{2}^{2}}\left(0, x_{2}\right)=y_{1}^{m} \frac{\partial^{2} u^{0}}{\partial x_{2}^{2}}\left(0, x_{2}\right),
\end{array}\right.
$$

In (42), we want $\partial_{x_{1}}\left\langle\sigma_{1}^{0}\right\rangle$ to appear, in order to absorb the (diverging) terms in $y_{1}^{m}$ in the matching condition (38). Do do so, we use (20) for $x_{1}<0$ and (16) for $x_{1}>0$ and we get

$$
\left\{\begin{aligned}
-\frac{\partial\left\langle\sigma_{1}^{0}\right\rangle}{\partial x_{1}}\left(0^{-}, x_{2}\right) & =\langle 1 / a\rangle^{-1} \frac{\partial^{2} u^{0}}{\partial x_{2}^{2}}\left(0, x_{2}\right)+\langle b\rangle u^{0}\left(0, x_{2}\right), \\
-\frac{\partial\left\langle\sigma_{1}^{0}\right\rangle}{\partial x_{1}}\left(0^{+}, x_{2}\right) & =\frac{\partial^{2} u^{0}}{\partial x_{2}^{2}}\left(0, x_{2}\right)+u^{0}\left(0, x_{2}\right),
\end{aligned}\right.
$$

whence

$\int_{Y} \mathrm{~d} \mathbf{y}\left[\frac{\partial^{2} u^{0}}{\partial x_{2}^{2}}\left(0, x_{2}\right) \frac{\tilde{a}(\mathbf{y}) / a\left(y_{2}\right)}{\langle 1 / a\rangle}+\tilde{b}(\mathbf{y}) u^{0}\left(0, x_{2}\right)\right]=-y_{1}^{m}\left[\frac{\partial\left\langle\sigma_{1}^{0}\right\rangle}{\partial x_{1}}\left(0^{+}, x_{2}\right)+\frac{\partial\left\langle\sigma_{1}^{0}\right\rangle}{\partial x_{1}}\left(0^{-}, x_{2}\right)\right]$

It is now sufficient to use (40), (41) and (44) in (39), to get the jump condition

$$
\llbracket\left\langle\sigma_{1}^{1}\right\rangle \rrbracket=-\mathcal{C} \frac{\partial^{2} u^{0}}{\partial x_{2}^{2}}\left(0, x_{2}\right), \quad \text { with } \mathcal{C} \equiv \int_{\mathbf{Y}} \mathrm{d} \mathbf{y} T_{2}^{(2)}(\mathbf{y}),
$$

and we used that $\int_{\mathrm{Y}} \mathrm{d} \mathbf{y} T_{2}^{(1)}(\mathbf{y})=0$ since $V^{(1)}$ is symmetric w.r.t. $y_{2}$. The constant $\mathcal{C}$ is the second interface parameters entering in the jump conditions.

2.3.4. Jump conditions and final homogenized problem. The jump conditions on $(\bar{u}, \overline{\boldsymbol{\sigma}})$ are deduced from (28), with (29),(30) and (37),(45), and read

$$
\llbracket \bar{u} \rrbracket=\varepsilon \mathcal{B}\left\langle\sigma_{1}^{0}\right\rangle\left(0, x_{2}\right), \quad \llbracket \bar{\sigma}_{1} \rrbracket=-\varepsilon \mathcal{C} \frac{\partial^{2} u^{0}}{\partial x_{2}^{2}}\left(0, x_{2}\right) .
$$

The above jump conditions define a homogenized problem which can be solved iteratively : first compute $\left(u^{0},\left\langle\boldsymbol{\sigma}^{0}\right\rangle\right)$ satisfying (16), (20) with (29) and (30) (compute also 
$\mathcal{B}$ and $\mathcal{C}$ ) and use the results to get the right hand-side term in (46); then, compute $(\bar{u}, \bar{\sigma})$ which approximate $\left(u^{\varepsilon}, \boldsymbol{\sigma}^{\varepsilon}\right)$ up to $O\left(\varepsilon^{2}\right)$. As discussed in [4], it is preferable to handle a unique problem and this is done by defining the fields $(\tilde{u}, \tilde{\boldsymbol{\sigma}})$ satisfying the following homogenized problem

$$
\begin{cases}\operatorname{div} \tilde{\boldsymbol{\sigma}}+\langle b\rangle \tilde{u}=0, \quad \tilde{\boldsymbol{\sigma}}=\left(\begin{array}{cc}
\langle a\rangle & 0 \\
0 & \langle 1 / a\rangle^{-1}
\end{array}\right) \nabla \tilde{u}, & x_{1}<0 \\
\operatorname{div} \tilde{\boldsymbol{\sigma}}+\tilde{u}=0, \quad \tilde{\boldsymbol{\sigma}}=\boldsymbol{\nabla} \tilde{u}, & x_{1}>0 \\
\llbracket \tilde{u} \rrbracket=\frac{\varepsilon \mathcal{B}}{2}\left[\tilde{\sigma}_{1}\left(0^{-}, x_{2}\right)+\tilde{\sigma}_{1}\left(0^{+}, x_{2}\right)\right], & \\
\llbracket \tilde{\sigma}_{1} \rrbracket=-\frac{\varepsilon \mathcal{C}}{2}\left[\frac{\partial^{2} \tilde{u}}{\partial x_{2}^{2}}\left(0^{-}, x_{2}\right)+\frac{\partial^{2} \tilde{u}}{\partial x_{2}^{2}}\left(0^{+}, x_{2}\right)\right], & \end{cases}
$$

and it is easy to see from (16), (27) and (46) that $\tilde{u}, \tilde{\boldsymbol{\sigma}}$ admit the expansions $\left\langle u^{0}\right\rangle+$ $\varepsilon\left\langle u^{1}\right\rangle,\left\langle\boldsymbol{\sigma}^{0}\right\rangle+\varepsilon\left\langle\boldsymbol{\sigma}^{1}\right\rangle$ up to $O\left(\varepsilon^{2}\right)$, thus the same expansion as $\left(u^{\varepsilon}, \boldsymbol{\sigma}^{\varepsilon}\right)$ up to $O\left(\varepsilon^{2}\right)$. Finally, coming back to the real space, in $\mathbf{X}=\mathbf{x} / k$ coordinate and with $U(\mathbf{X})=\tilde{u}(\mathbf{x})$, $\boldsymbol{\Sigma}(\mathbf{X})=k \tilde{\boldsymbol{\sigma}}(\mathbf{x}),(47)$ take the form

$$
\begin{cases}\operatorname{div} \boldsymbol{\Sigma}+\langle b\rangle k^{2} U=0, \quad \boldsymbol{\Sigma}=\left(\begin{array}{cc}
\langle a\rangle & 0 \\
0 & \langle 1 / a\rangle^{-1}
\end{array}\right) \boldsymbol{\nabla} U, & X_{1}<0 \\
\operatorname{div} \boldsymbol{\Sigma}+k^{2} U=0, \quad \boldsymbol{\Sigma}=\boldsymbol{\nabla} U, & X_{1}>0 \\
\llbracket U \rrbracket=\frac{h \mathcal{B}}{2}\left[\Sigma_{1}\left(0^{-}, X_{2}\right)+\Sigma_{1}\left(0^{+}, X_{2}\right)\right], & \\
\llbracket \Sigma_{1} \rrbracket=-\frac{h \mathcal{C}}{2}\left[\frac{\partial^{2} U}{\partial X_{2}^{2}}\left(0^{-}, X_{2}\right)+\frac{\partial^{2} U}{\partial X_{2}^{2}}\left(0^{+}, X_{2}\right)\right] . & \end{cases}
$$

The above problem, written for a single interface at $X_{1}=0$, correspond to the system (3)-(4) when two interfaces at $X_{1}= \pm e / 2$ are considered.

3. Scattering by an array of rectangular voids. In this section, we apply the previous analysis to rectangular voids or cracks, free of stresses (with Neumann conditions on their boundaries), periodically spaced in a homogeneous matrix being composed of the same elastic material than the substrate. In electromagnetism, this corresponds to a (perfect conducting) metallic array in a dielectric or in the air; in acoustics to an array of sound hard material in a fluid. We consider the scattering of an incident plane wave $U^{\text {inc }}$ arriving from $X_{1}<0$ and hitting the array at oblique incidence $\theta$ (Figure 5), whence

$$
U^{\mathrm{inc}}(\mathbf{X})=e^{\mathrm{i} k\left(X_{1}+e / 2\right) \cos \theta+\mathrm{i} k X_{2} \sin \theta}
$$

In the actual problem, the solution is sought in the substrate where the Helmholtz equation apply with Neumann boundary conditions on the boundaries of the voids occupying the subdomains $\Omega_{i}, i=1 \ldots N_{v}$ and $\Omega_{v}=\underset{i}{\cup} \Omega_{i}$. The problem is solved in 

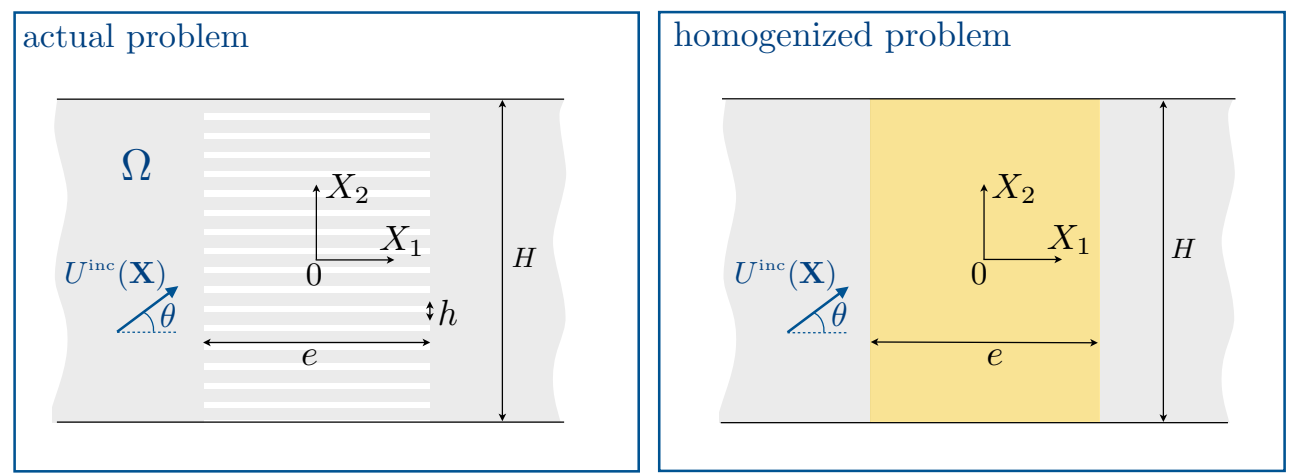

FIGURE 5. Actual problem of the scattering of a plane wave at oblique incidence $\theta$ on an array of rectangular voids; the problem is solved numerically. The homogenized problem involves a slab of same thickness e filled with a homogeneous anisotropic material (the wave equation being (51)); jump conditions (4) apply at $X_{1}= \pm e / 2$.

$\Omega \backslash \Omega_{v}$ with $\Omega=\left\{\left(X_{1}, X_{2}\right) \in \mathbb{R} \times(-H / 2, H / 2)\right\}$ and reads

$$
\begin{cases}\Delta U+k^{2} U=0, & \text { in } \Omega \backslash \Omega_{v}, \\ \nabla U \cdot \mathbf{n}=0, & \text { on } \partial \Omega_{i}, i=1 \ldots N_{v}, \\ \lim _{X_{1} \rightarrow \pm \infty}\left[\frac{\partial}{\partial X_{1}}\left(U-U^{\mathrm{inc}}\right) \mp \mathrm{i} k \cos \theta\left(U-U^{\mathrm{inc}}\right)\right]=0, & \\ U\left(X_{1}, \frac{H}{2}\right)=e^{\mathrm{i} k H \sin \theta} U\left(X_{1},-\frac{H}{2}\right), & X_{1} \in \mathbb{R}, \\ \frac{\partial U}{\partial X_{2}}\left(X_{1}, \frac{H}{2}\right)=e^{\mathrm{i} k H \sin \theta} \frac{\partial U}{\partial X_{2}}\left(X_{1},-\frac{H}{2}\right), & X_{1} \in \mathbb{R} .\end{cases}
$$

The conditions at $X_{1} \rightarrow \pm \infty$ are the radiation conditions required to select an outgoing scattered waves $\left(U-U^{\text {inc }}\right)$ in the low frequency regime, namely for $k<2 \pi / h$ (if not the case, the radiation condition should to be modified, see [2]). In the case where $H=n h$, with $n$ an integer (and we shall consider that this is the case), the last condition is referred to as the condition of pseudo-periodicity or the Floquet condition, which applies for the incident wave and for the total field [13]. The actual problem is solved numerically using a multimodal method which reduces to the determination of a set of scalar coefficients for $\left|X_{1}\right|<e / 2$ and for $\left|X_{1}\right|>e / 2$ (see subsection S2.2.1). In the following, the computed solution $U^{\text {num }}$ is the reference solution.

3.1. Solutions of the homogenized problems at the first and at the second orders. We shall see that the homogenized problems at the first two orders can be solved exactly. Voids or cracks correspond to the limiting case $a=0=b$ (leading to Neumann boundary condition at the boundary with any other material). Next, with $a=1=b$ in the substrate, and with $\varphi$ the filling fraction of the substrate in the layers, the equivalent medium has bulk parameters $\langle a\rangle=\langle b\rangle=\varphi$ and $\langle 1 / a\rangle^{-1}=0$, whence the homogenized wave equation (3) becomes

$$
\operatorname{div} \boldsymbol{\Sigma}+\varphi k^{2} U=0, \quad \boldsymbol{\Sigma}=\left(\begin{array}{cc}
\varphi & 0 \\
0 & 0
\end{array}\right) \nabla U
$$


It follows that the homogenized problems read

$$
\begin{cases}\frac{\partial^{2} U}{\partial X_{1}^{2}}+k^{2} U=0, & \text { for }\left|X_{1}\right|<\frac{e}{2}, \\ \Delta U+k^{2} U=0, & \text { for }\left|X_{1}\right|>\frac{e}{2} \\ \text { Jump conditions }(4), & \text { at } X_{1}= \pm \frac{e}{2}, \\ \lim _{X_{1} \rightarrow \pm \infty}\left[\frac{\partial}{\partial X_{1}}\left(U-U^{\mathrm{inc}}\right) \mp \mathrm{i} k \cos \theta\left(U-U^{\mathrm{inc}}\right)\right]=0, & \\ U\left(X_{1}, \frac{H}{2}\right)=e^{\mathrm{i} k H \sin \theta}\left(X_{1},-\frac{H}{2}\right), & X_{1} \in \mathbb{R}, \\ \frac{\partial U}{\partial X_{2}}\left(X_{1}, \frac{H}{2}\right)=e^{\mathrm{i} k H \sin \theta} \frac{\partial U}{\partial X_{2}}\left(X_{1},-\frac{H}{2}\right), & X_{1} \in \mathbb{R} .\end{cases}
$$

In the following, we refer to the homogenized problems at first and at second orders for the values of $(\mathcal{B}, \mathcal{C})$ entering in the jump conditions $(4)$; at first order, $(\mathcal{B}, \mathcal{C})=(0,0)$ and at second order,

$$
\mathcal{B}=-\frac{1}{\pi} \log \sin \left(\frac{\pi \varphi}{2}\right), \quad \mathcal{C} \simeq \frac{\pi}{16} \varphi^{2},
$$

(see subsection $\mathrm{S} 2.1$ ).

The solution of (52) with (49) is of the form

$$
\begin{cases}X_{1}<-\frac{e}{2}, & U(\mathbf{X})=\left[e^{\mathrm{i} k\left(X_{1}+e / 2\right) \cos \theta}+R e^{-\mathrm{i} k\left(X_{1}+e / 2\right) \cos \theta}\right] e^{\mathrm{i} k X_{2} \sin \theta}, \\ \left|X_{1}\right|<\frac{e}{2}, & U(\mathbf{X})=\left[a e^{\mathrm{i} k X_{1}}+b e^{-\mathrm{i} k X_{1}}\right] e^{\mathrm{i} k X_{2} \sin \theta}, \\ X_{1}>\frac{e}{2}, & U(\mathbf{X})=T e^{\mathrm{i} k\left(X_{1}-e / 2\right) \cos \theta+\mathrm{i} k X_{2} \sin \theta},\end{cases}
$$

with $(R, T, a, b)$ given by the jump conditions (4). Indeed, applying (4) to (54), we find, for any $(k, \theta)$ values characterizing the incident wave, a set $(R, T, a, b)$ such that $U$ is solution of the homogenized problems. In particular, the scattering coefficients $(R, T) \mathrm{read}$

$$
\left\{\begin{array}{l}
R=-\frac{z_{1}^{*} z_{2}{ }^{*} e^{\mathrm{i} k e}-z_{1} z_{2} e^{-\mathrm{i} k e}}{z_{1}{ }^{2} e^{\mathrm{i} k e}-z_{2}^{2} e^{-\mathrm{i} k e}} \\
T=\frac{\left|z_{1}\right|^{2}-\left|z_{2}\right|^{2}}{z_{1}^{* 2} e^{\mathrm{i} k e}-z_{2}^{2} e^{-\mathrm{i} k e}}
\end{array}\right.
$$

$$
\left\{\begin{array}{l}
z_{1} \equiv\left(1-\frac{\cos \theta}{\varphi}\right)+\mathrm{i} k h\left(\mathcal{B} \cos \theta+\mathcal{C} \frac{\sin ^{2} \theta}{\varphi}\right)-(k h)^{2} \sin ^{2} \theta \frac{\mathcal{B C}}{4}\left(1+\frac{\cos \theta}{\varphi}\right), \\
z_{2} \equiv\left(1+\frac{\cos \theta}{\varphi}\right)-\mathrm{i} k h\left(\mathcal{B} \cos \theta-\mathcal{C} \frac{\sin ^{2} \theta}{\varphi}\right)+(k h)^{2} \sin ^{2} \theta \frac{\mathcal{B C}}{4}\left(1+\frac{\cos \theta}{\varphi}\right) .
\end{array}\right.
$$

Obviously, for $\mathcal{B}=\mathcal{C}=0$, the jump conditions (4) simplify to the continuities of $U$ and $\boldsymbol{\Sigma} \cdot \mathbf{n}$ and we recover the usual expressions of the scattering coefficients given by the homogenization at the first order, with $\cos \theta / \varphi$ the effective impedance mismatch between the two media [12]. Finally, the derivation of $(a, b)$ is straightforward and together with $(R, T)$ can be used in (54) to calculate the homogenized wavefield $U(\mathbf{X})$. 
3.2. Numerical validation of the homogenized solutions. We shall inspect the validity of the homogenized solution up to the first and second orders for an incident plane wave at normal and at oblique incidences. To begin with, we report the fields $U^{\text {num }}$ calculated numerically and the fields $U$ of the homogenized solutions, (54), with (55)-(56), for $\varphi=0.5$ (Figure 6) and $\varphi=0.9$ (Figure 7); in both cases, $k h=1$, $e / h=10$ and $\theta=\pi / 3$. The improvement in the solutions given by the homogenization at the second order, compared to the ones given by the first order, is visible to the naked eye. Defining $\Delta U \equiv\left|U-U^{\text {num }}\right| /\left|U^{\text {num }}\right|$ (for $\left|X_{1}\right|>e / 2$ and with $|$.$| the L^{2}$ norm), we get a discrepancy of $10 \%(\varphi=0.5)$ and $20 \%(\varphi=0.9)$ for the first order homogenization, and of $0.7 \%-0.6 \%$ for the second order homogenization. It is worth noting that a very small error is found even at this relatively large $k h=1$ value.
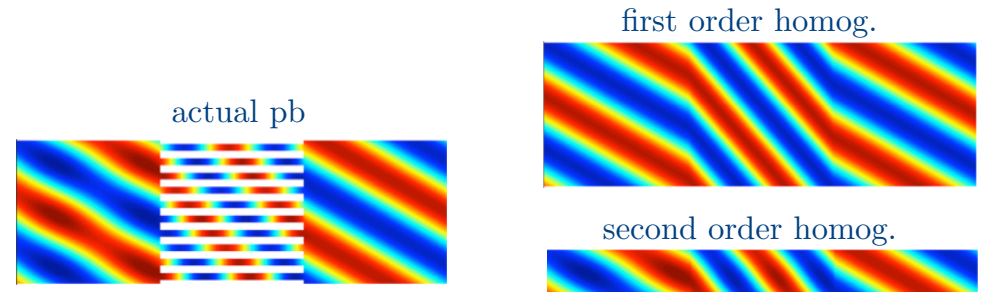

second order homog.

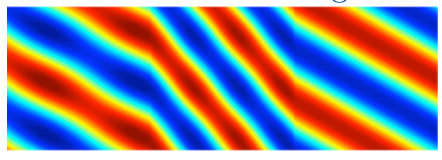

Figure 6. Scattering of a plane wave at incidence $\theta=\pi / 3$ and $k h=1$ on a array made of rectangular voids $(e / h=10$ and $\varphi=0.5)$. The solution $U^{\text {num }}$ in the actual problem is calculated numerically; on the right, the fields $U$ given by the homogenization at the first and at the second orders.

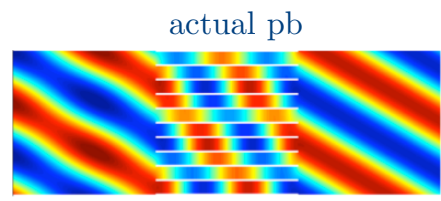

first order homog.

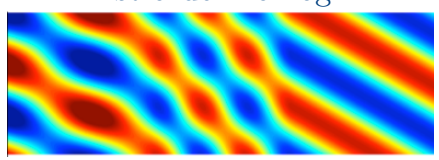

second order homog.

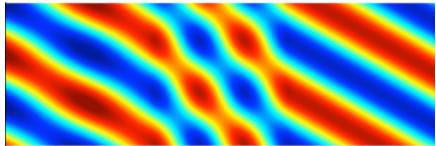

FiguRE 7. Same representation as in Figure 6 with $\varphi=0.9$.

3.2.1. Waves at normal incidence. We first inspect the case $\theta=0$, for which the homogenizations, at first and second orders, impose $\llbracket \boldsymbol{\Sigma} \rrbracket \cdot \mathbf{n}=0$ in (4) (because from (52), $U\left(X_{1}, X_{2}\right)=U\left(X_{1}, 0\right)$ does not depend on $\left.X_{2}\right)$, thus only the influence of $\mathcal{B}$ in the jump condition for the displacement is regarded (see also (55) with (56)). We report in Figure 8 the spectra of the transmission as a function of $k h$ and $e / h$ (with $\varphi=0.1$ and $\theta=0$ ) and in Figure 9 the corresponding errors $\Delta T=\left|T^{\text {num }}-T\right| /\left|T^{\text {num }}\right|$ (for the homogenizations at the first and at the second orders). We considered $k h \in[0,2 \pi]$, $k h=2 \pi$ corresponding to the cut-off frequency above which a second propagating mode exists in the actual problem.

In Figure 9, errors smaller than $1 \%$ appear in dark blue and errors grater than 


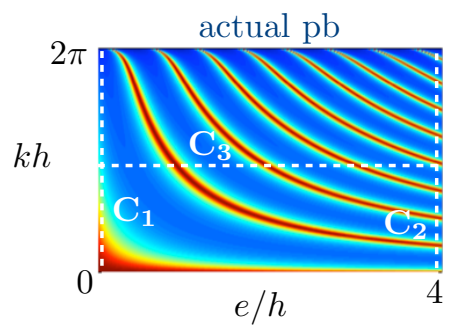

first order homog.

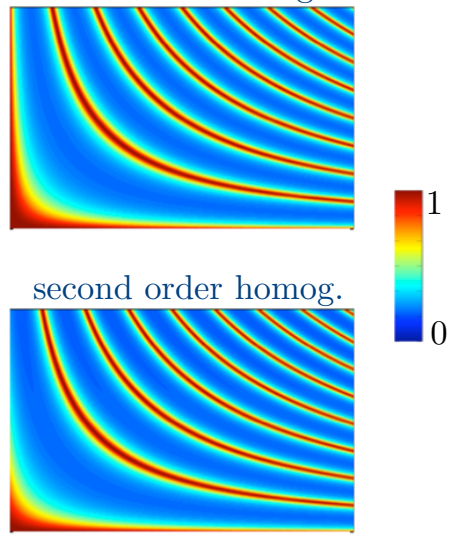

FIGURE 8. Transmission coefficient as a function of $e / h$ and of the frequency $k h ; \varphi=0.1$ and $\theta=0$ have been considered. On the left, in the actual problem, $\left|T^{\mathrm{num}}\right|$ calculated numerically, and on the right, $|T|$ given by the first and second order homogenizations, (55), with (56).

$100 \%$ in dark red. The range of validity of the homogenized solution is significantly increased when using the second order homogenization; on the one hand, intermediate frequencies become accessible (the error is smaller than $4 \%$ for $k h<\pi$ in the whole range of $e / h$ at second order, it is of $50 \%$ on average at first order) ; on the other hand, the first order homogenization predicts erroneously perfect transmissions for vanishing thicknesses $e / h$, while going up to the second order restores the actual scattering properties of an array of flat voids. This is related to the discussion presented in Ref. [7] in the context of electromagnetic waves. In this reference, an effective medium approach at second order is presented assuming the continuities of the displacement and of the normal stress (instead of our jump conditions); the analysis concludes that the effective bulk parameter $a$ has to be dependent on the thickness (in this reference, $a$ denotes the effective permittivity for electromagnetic waves).
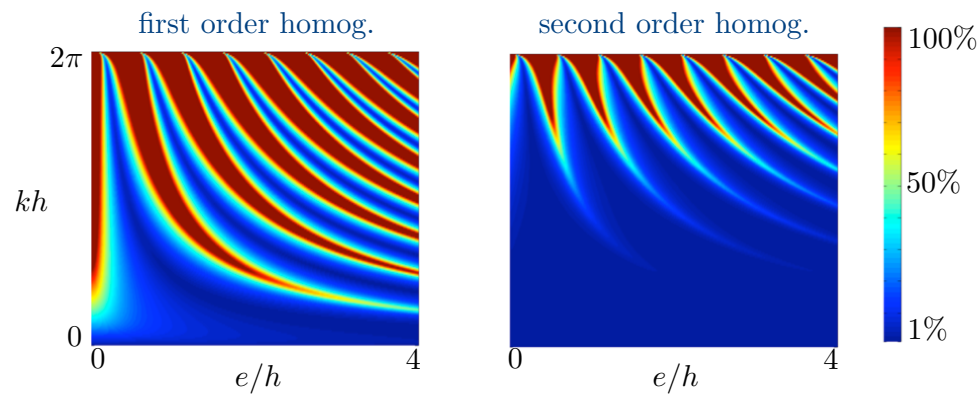

FIGURE 9. Errors $\Delta T$ on the transmission coefficient in the homogenization problems (at first and at second orders) as a function of $e / h$ and $k h(\varphi=0.1$ and $\theta=0)$. Errors below $1 \%$ appear in dark blue and errors larger than 100\% appear in dark red.

More specifically, we inspect (i) the profiles of $\left|T^{\text {num }}\right|$ (blue symbols) and its homogenized counterparts $|T|$ at first order (grey lines) and at second order (black lines) as a function of $k h$ for $e / h=0.05$ and $e / h=4$ (Figure 10). For a small thickness $\left(\mathrm{C}_{1}\right.$ profile from Figure 8$)$, the homogenization at first order largely overestimates the transmission while including the jump conditions (4) at second order recovers 

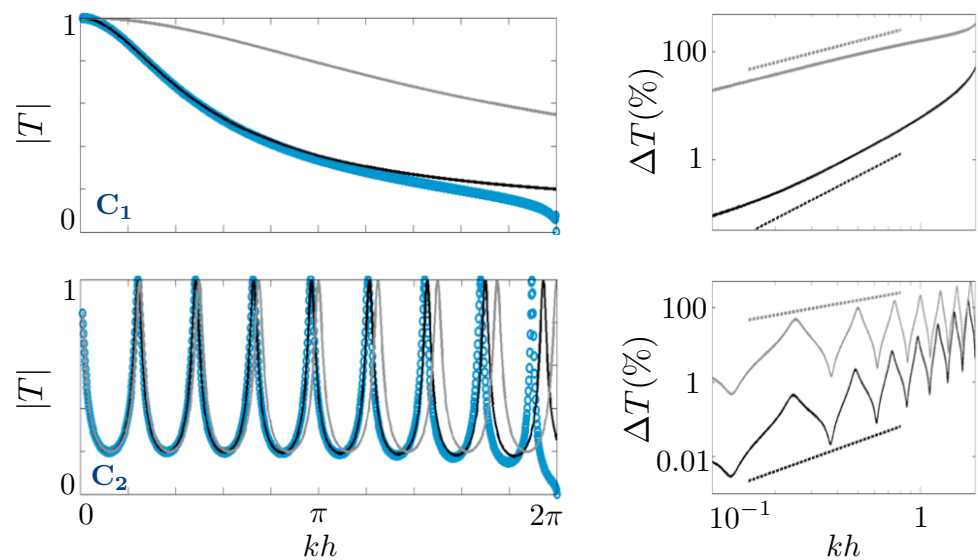

FIGURE 10. Transmission coefficients $\left|T^{\text {num }}\right|$ and $|T|$ as a function of $k h$ and corresponding error $\Delta T$ of the homogenized predictions. $C_{1}$ profile for $e / h=0.05$ and $C_{2}$ for $e / h=4\left(\left|T^{\mathrm{num}}\right|\right.$ : blue symbols and $|T|$ : grey lines at first order and black lines at second order). $\Delta T$ are shown in $\%$, dotted lines are guidelines with slopes kh (grey) and $(k h)^{2}$ (black).

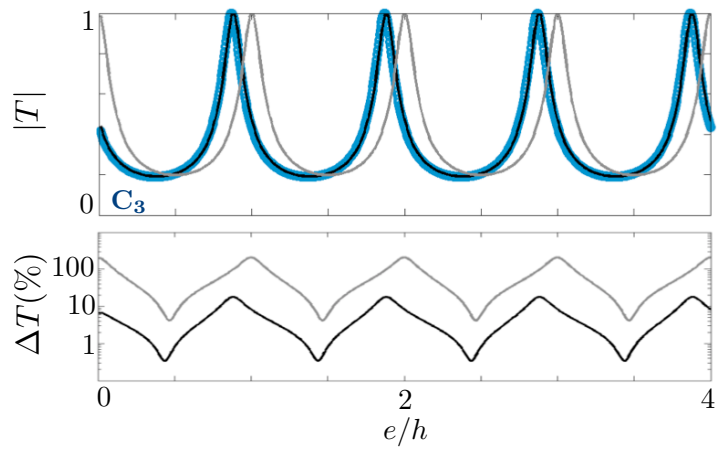

Figure 11. Transmission coefficient and errors as a function of e/h for $k h=\pi$ ( $C_{3}$ profile from Figure 8). Same representation as in Figure 10.

the actual transmission of the array; for larger array $\left(\mathrm{C}_{2}\right.$ profile $)$ the first order homogenization is valid for small $k h$ and going up to the second order allows us to not periodic w.r.t. $k h$ (the $k h$-distance between two perfect transmissions decreases as $k h$ increases); from (55), this tendency is correctly captured by $T$ at second order, T at first order appears to be erroneously periodic, with $2 \pi h / e$ period. Finally, order

(ii) the variations of $\left|T^{\text {num }}\right|$ and $|T|$ (and the corresponding errors $\Delta T$ ) as a function of $e / h$ for $k h=\pi$ are reported in Figure $11\left(\mathrm{C}_{3}\right.$ profile from Figure 8). Amusingly, the large error in the first order homogenization appears to be a direct consequence of the "initial" error for vanishing $e / h$; there, $|T|=1$ is erroneously found at the first order, and the periodicity of $T$ w.r.t $e / h$ imposes the same error to occur for any $e / h$ (the periodicity of $T$ w.r.t. $e / h$ is, from (55), $2 \pi / k h$, thus equals 2 in the present case). 
3.2.2. The case of oblique incidence. In the case of oblique incidence $(\theta=$ $\pi / 3$ is considered in this section), the difference between the homogenized solutions at first and second orders involves the two interface parameters $(\mathcal{B}, \mathcal{C})$. We report the same sequence of figures as for the normal incidence; with $k h \in[0,2 \pi]$, the frequency range includes $k h>2 \pi /(1+\sin \theta) \simeq 1.07 \pi$ where two modes are propagating (complete extinction of the first mode is observed at this cut-off frequency, Figure 12 and Figure 14, corresponding to the Wood anomaly [10]). This range is outside the range of validity of any homogenization approach since mode coupling is not possible at an equivalent flat boundary [10].
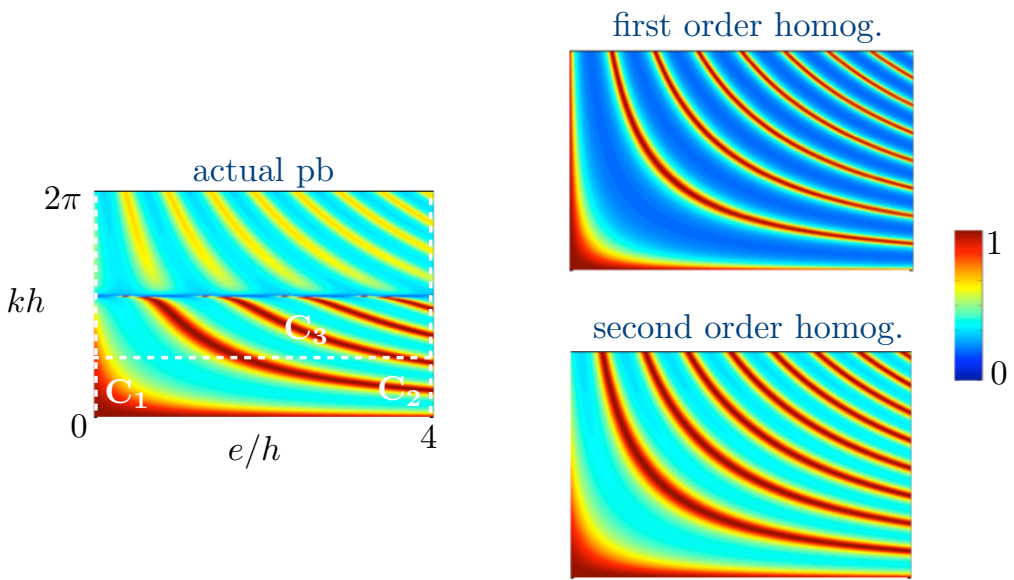

Figure 12. Same representation as Figure 8 for $\theta=\pi / 3$.
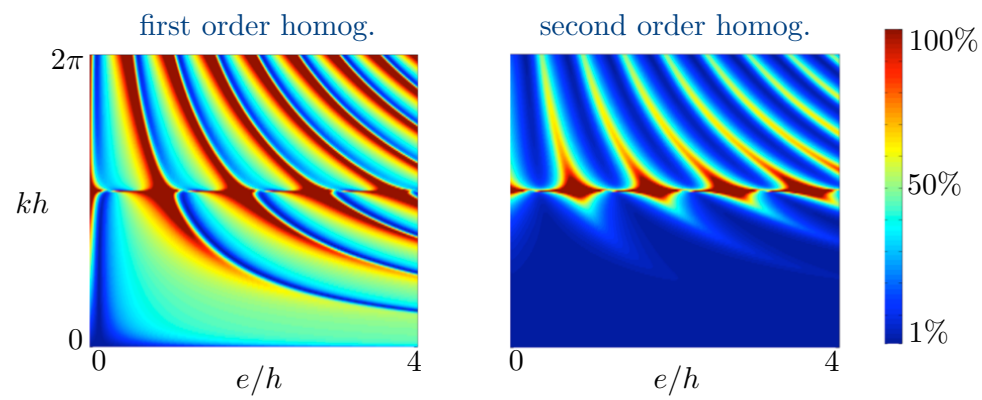

FiguRE 13. Same representation as Figure 9 for $\theta=\pi / 3$.

Inspecting Figure 12 to Figure 15, we recover in general the same tendencies as those observed at normal incidence. On average, for $k h<\pi / 2$, the error in the transmission coefficient is smaller than $1 \%$ in the whole range of $e / h$ at second order, it is of $40 \%$ on average at first order. Also, the first order homogenization underestimates the scattering properties of the structure when $e / h$ goes to zero $\left(\mathrm{C}_{1}\right.$ profile in Figure 14 and Figure 15). Reversely for larger $e / h$, and this is more surprising, it overestimates the scattering properties (with $|T|$ significantly smaller than $\left|T^{\text {num }}\right|$ ) even for small $k h$ value (from Figure 12 and the $\mathrm{C}_{2}$ profile in Figure 14). Thus, in general, the improvement in the second order homogenized solution is even more significant at an oblique incidence. 

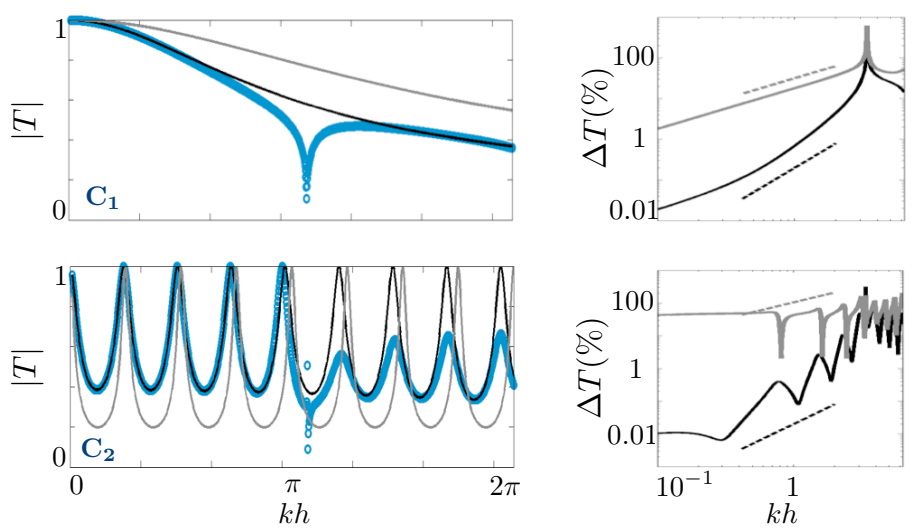

FIGURE 14. Same representation as Figure 10 for $\theta=\pi / 3$.

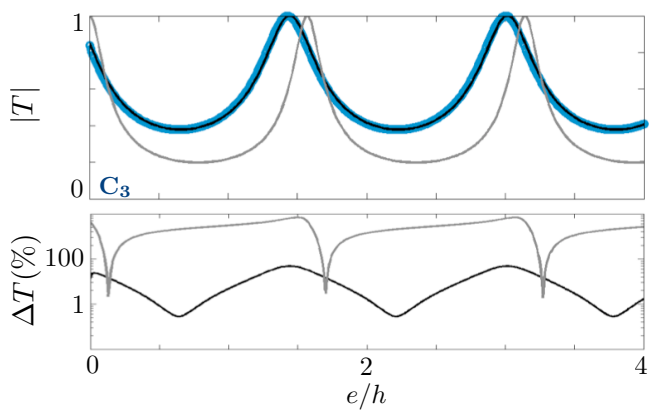

Figure 15. Transmission coefficient and errors as a function of e/ $h$ for $k h=0.6 \pi\left(C_{3}\right.$ profile from Figure 12). Same representation as in Figure 10.

4. Concluding remarks. The homogenization that we have presented explicitly accounts for the finite size $e / h$ of stratified structures. At first and at second orders, this makes effective bulk parameters to appear which are simply averages of the bulk parameters in each layer and these effective bulk parameters enter in the homogenized wave equation. Note the simplicity happening in the case of stratified media : (i) the bulk parameters are found without solving cell problems, and this is well known, (ii) the same effective wave equation is found at first and at second orders, and this is less known. Note also that this simplicity would be lost in the case of periodic media with a more complex unit cell. Finally, the most significant improvement in the presented approach is the derivation of jump conditions involving effective interface parameters when accounting for the finite thickness of the structure. More usually, the continuity of the displacement and of the normal stress are assumed, and this is what we recover at first order. Going up to second order allows us to establish jump conditions for these fields and the improvement in the homogenized result has been exemplified.

Acknowledgments. J.-J.M. thanks the support of the French Agence Nationale de la Recherche (ANR), under grant Aramis (ANR-12-BS01-0021) "Analysis of Robust Asymptotic Methods In numerical Simulation in mechanics". A.M. thank the support of the LABEX WIFI (Laboratory of Excellence within the French Program "Investments for the Future") under references ANR-10-LABX-24 and ANR- 


\section{0-IDEX-0001-02 PSL*.}

The authors thank the anonymous reviewers for their insightful comments and suggestions.

[1] R. Abdelmoula and J.-J. Marigo, The effective behavior of a fiber bridged crack, Journal of the Mechanics and Physics of Solids, 48 (2000), pp. 2419-2444.

[2] G. Bouchitté AND D. FelbacQ, Homogenization of a wire photonic crystal : the case of small volume fraction, SIAM Journal on Applied Mathematics, 66 (2006), pp. 2061-2084.

[3] D. Cioranescu and P. Donato, An introduction to homogenization, volume 17 of oxford lecture series in mathematics and its applications, The Clarendon Press Oxford University Press, New York, 4 (1999), p. 118.

[4] M. David, J.-J. Marigo, And C. Pideri, Homogenized interface model describing inhomogeneities located on a surface, Journal of Elasticity, 109 (2012), pp. 153-187.

[5] B. Delourme, Modèles et asymptotiques des interfaces fines et périodiques en électromagnétisme, $\mathrm{PhD}$ thesis, Université Pierre et Marie Curie-Paris VI, 2010.

[6] B. Delourme, H. Haddar, And P. Joly, Approximate models for wave propagation across thin periodic interfaces, Journal de mathématiques pures et appliquées, 98 (2012), pp. 28-71.

[7] P. Lalanne and D. Lemercier-Lalanne, Depth dependence of the effective properties of subwavelength gratings, JOSA A, 14 (1997), pp. 450-459.

[8] M. Lapine, R. McPhedran, and C. Poulton, Slow convergence to effective medium in finite discrete metamaterials, Physical Review B, 93 (2016), p. 235156.

[9] J.-J. Marigo and A. Maurel, Homogenization models for thin rigid structured surfaces and films, The Journal of the Acoustical Society of America, 140 (2016), pp. 260-273.

[10] A. Maurel, S. FÉLIX, J.-F. Mercier, AND A. OURIR, Effective birefringence to analyze sound transmission through a layer with subwavelength slits, Comptes Rendus Mécanique, 343 (2015), pp. 612-621.

[11] A. Maurel, J.-J. Marigo, And A. Ourir, Homogenization of ultrathin metallo-dielectric structures leading to transmission conditions at an equivalent interface, The Journal of the Optical Society of America B, 33 (2016), pp. 947-956.

[12] J.-F. Mercier, M. Cordero, S. Félix, A. Ourir, And A. Maurel, Classical homogenization to analyse the dispersion relations of spoof plasmons with geometrical and compositional effects, in Proc. R. Soc. A, vol. 471, The Royal Society, 2015, p. 20150472.

[13] R. Petit, A tutorial introduction, in Electromagnetic Theory of Gratings, Springer, 1980, pp. $1-52$.

[14] S. RYTov, Electromagnetic properties of a finely stratified medium, Soviet Physics JETP-USSR, 2 (1956), pp. 466-475. 
Annexe A. The case of vanishing slab thicknesses. The validity of the homogenization of stratified media with finite size has been exemplified in this paper for varying $e / h$. In fact, the case of vanishing thicknesses has to be inspected more carefully. Indeed, we end with jump conditions reflecting the behavior of the evanescent field at each boundary of the equivalent slab, and these boundary layers have been assumed to be independent. In fact, for very small thicknesses, the two boundary layers may interact. In this case, the bulk problem is meaningless and the elementary problems have to include the whole structure; doing so, jump conditions across an equivalent interface are found (and the bulk behavior is disregarded), see [9, 11]. We obtained in this case

$$
\left\{\begin{array}{l}
R^{\mathrm{int}}=-\frac{1}{2}\left[\frac{Z_{1}^{*}}{Z_{1}}+\frac{Z_{2}^{*}}{Z_{2}}\right], \\
T^{\mathrm{int}}=\frac{1}{2}\left[\frac{Z_{1}^{*}}{Z_{1}}-\frac{Z_{2}^{*}}{Z_{2}}\right],
\end{array}\right.
$$

$$
\left\{\begin{array}{l}
Z_{1}=1-\mathrm{i} k h \cos \theta\left(\mathcal{B}+\frac{e}{2 \varphi}\right), \\
Z_{2}=-\mathrm{i} \cos \theta-k h\left(\frac{e \varphi}{2 h}+\mathcal{C} \sin ^{2} \theta\right),
\end{array}\right.
$$

with $(\mathcal{B}, \mathcal{C})$ in $(53)$. Here, we illustrate the limitation of the present homogenization in the light of the size of the boundary layers in the actual problem.

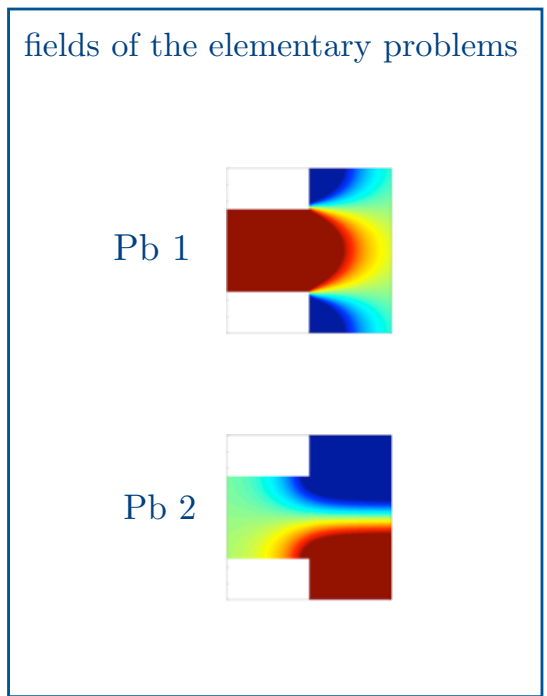

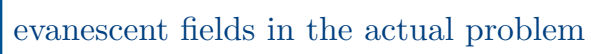
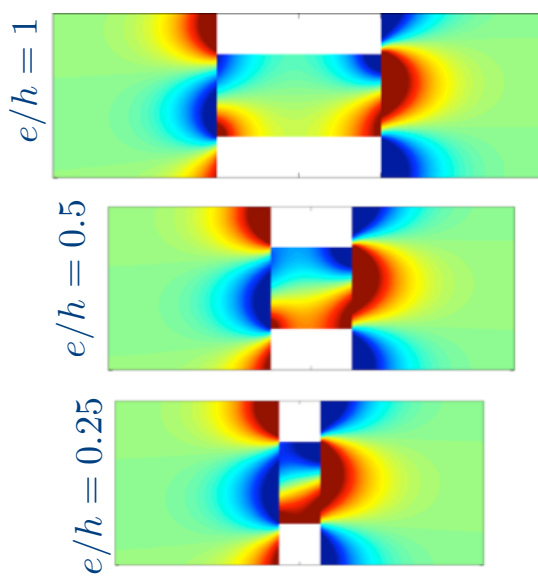

FIGURE 16. Boundary layers in the static elementary problems and evanescent fields in the actual problems for $\varphi=0.5$. In the actual problem, we considered $k h=1$ and $\theta=\pi / 4$.

In Figure 16, we reported (i) the elementary solutions $V^{(1,2)}$ of (S9) and (S17) for $\varphi=0.5$ (for $V^{(1)}$, the external load is $\left(y_{1}\right)$ and it has been removed to exhibit the resulting boundary layer), (ii) the evanescent fields in the actual problem for $k h=1$ and varying $e / h=1,0.5$ and 0.25 (the total wavefields are continuous but the evanescent fields are not). Qualitatively, for $e / h=1$, it is visible that the evanescent 

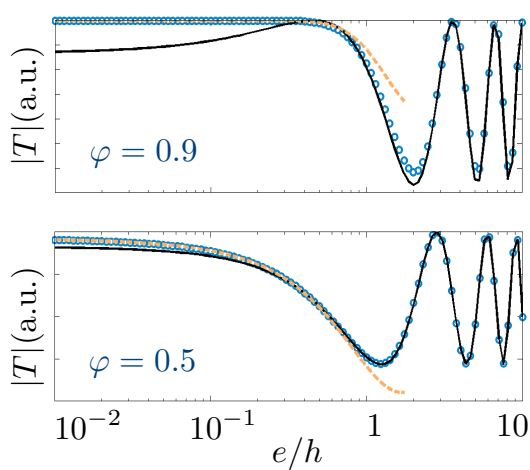
the boundary layer effects.

fields in the actual problem ressemble (by parts) to the fields in the elementary problems. This is quite natural if one remembers that the evanescent field in the actual problem can be decomposed in modes with $y_{1}$ dependance being of the form $e^{ \pm \mathrm{i} k_{n} y_{1}}$, and $k_{n}=\sqrt{k^{2}-(n \pi / h)^{2}} \sim \mathrm{i}(n \pi / h)$ for $y_{1}>0, k_{n}=\sqrt{k^{2}-(n \pi / h \varphi)^{2}} \simeq \mathrm{i}(n \pi / h \varphi)$ for $y_{1}<0$, thus involving at the dominant order the same $y_{1}$ dependences as in the static problems (see subsection S2.2.1). Decreasing $e$ result in the interaction of the evanescent fields within the array, or in other words the whole array is concerned by

Quantitatively, Figure 17 shows $T^{\text {num }}$ and $T$ (at second order) and the resulting error $\Delta T$ as a function of $e / h$ in logscale up to $e / h=10^{-2}$ (and $\varphi=0.1,0.5$ and $0.9)$. It is visible that the error significantly increases for $e / h<1$, and the increase is more significant for larger $\varphi$; to estimate more carefully the critical thickness below which the present homogenization is not valid anymore (or say less accurate), one should consider the influence of $\varphi$ ( $\varphi$ being a measure of the size of boundary layer, to be compared to $e / h$ the array thickness in $\mathbf{y}$-coordinates). We report in dotted lines the transmission coefficient $T^{\text {int }}$ given by the interface homogenization (57)-(58) (from [9]. As expected, this homogenization which accounts for the boundary layers in the whole array, is more accurate for vanishing thickness.

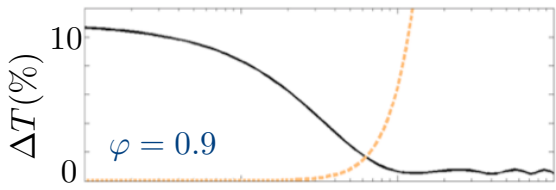

FIGURE 17. Transmission coefficient $\left|T^{\mathrm{num}}\right|$ (blue symbols) and $|T|$ at second order (black lines) as a function of e/ $h$ for $\varphi=0.9$ and 0.5 , and corresponding errors $\Delta T$. The errors increase significantly for roughly $e / h<1$. Dotted orange lines show the transmission coefficient $\left|T^{\mathrm{int}}\right|$ obtained using interface homogenization (57)-(58) (from [9]). 